\title{
An Effective Technique for Enhancing an Intrauterine Catheter Fetal Electrocardiogram
}

\author{
Steven L. Horner \\ Department of Electrical Engineering, Bucknell University, Lewisburg, PA 17837, USA \\ Email:shorner@bucknell.edu \\ William M. Holls III \\ University of Illinois, Provena Covenant Medical Center, 1400 West Park Street, Urbana, IL 61801, USA \\ Email:wholls@uiuc.edu
}

Received 11 August 2001 and in revised form 20 August 2002

\begin{abstract}
Physicians can obtain fetal heart rate, electrophysiological information, and uterine contraction activity for determining fetal status from an intrauterine catheters electrocardiogram with the maternal electrocardiogram canceled. In addition, the intrauterine catheter would allow physicians to acquire fetal status with one noninvasive to the fetus biosensor as compared to invasive to the fetus scalp electrode and intrauterine pressure catheter used currently. A real-time maternal electrocardiogram cancellation technique of the intrauterine catheters electrocardiogram will be discussed along with an analysis for the methods effectiveness with synthesized and clinical data. The positive results from an original detailed subjective and objective analysis of synthesized and clinical data clearly indicate that the maternal electrocardiogram cancellation method was found to be effective. The resulting intrauterine catheters electrocardiogram from effectively canceling the maternal electrocardiogram could be used for determining fetal heart rate, fetal electrocardiogram electrophysiological information, and uterine contraction activity.
\end{abstract}

Keywords and phrases: fetal electrocardiogram, intrauterine catheter, scalp electrode, maternal electrocardiogram.

\section{INTRODUCTION}

The invasive scalp electrode has proven to be a reliable technique for acquiring the fetal electrocardiogram (FECG) during delivery [1]. From the FECG, physicians can determine fetal heart rate (HR). The HR can then be used to monitor the status of the fetus. However, the scalp electrode is invasive to the mother and fetus, and also has limitations. One limitation includes risk of viral infection from the mother since there can be blood-to-blood contact. Furthermore, the scalp electrode cannot be modified with a pressure transducer to monitor maternal contractions. Currently, a scalp electrode and intrauterine pressure catheter are placed to monitor fetal HR and maternal contraction information, respectively [2].

Another FECG monitoring technique used during delivery includes the intrauterine catheter (IC) $[2,3]$. The IC can be used to monitor fetal HR and electrophysiological information during delivery. Since the IC is noninvasive to the fetus but invasive to the mother, the catheter makes a nice alternative from the scalp electrode. Compared to the noninvasive abdominal-wall approach, the catheter is placed in close proximity to the fetus and can touch the fetus in some places $[2,4]$. The adjacency allows for an increased probability of obtaining a FECG with a favorable signalto-noise ratio (SNR). In addition, the FECG of the IC can be combined with the intrauterine pressure catheter. These combined catheters could perform the tasks of the scalp electrode and intrauterine pressure catheter with only inserting one biosensor in the uterus [2,3].

The objective of this paper is to develop an effective technique for maternal electrocardiogram (ECG) cancellation of the IC's ECG. The goals that fulfill this objective are as follows:

(i) develop a method for canceling the maternal ECG of an IC's ECG;

(ii) ascertain the method's effectiveness for maternal ECG cancellation;

(iii) conclude from goal two whether the method has been effective for canceling the maternal ECG of the IC's ECG.

Since acquiring electrophysiological information from an IC usually requires FECG SNR enhancement, a standard FECG averaging algorithm has also been included and is incorporated with the analysis of the maternal ECG cancellation method [5]. 


\section{LITERATURE REVIEW}

Two previously reported intrauterine catheter techniques are discussed in this section along with the research contribution of this paper $[2,3]$. Before the previously reported IC techniques are described, a mathematical description of a biopotential signal obtained from the periphery or internally of a pregnant woman will be presented.

\subsection{ECG signal description}

A sampled measured signal from the periphery or internally of a pregnant woman can be described as

$$
\begin{aligned}
S_{k}(n)= & \operatorname{Md}_{k}(n) M_{k}(n)+F_{k}(n)+N_{k, \mathrm{EMG}}(n) \\
& +N_{k, 60 \mathrm{~Hz}}(n)+N_{k, \text { amp }}(n)+N_{k, \text { therm }}(n)+N_{k, \text { art }}(n),
\end{aligned}
$$

where $k$ is the ECG lead or channel number, $M_{k}(n)$ is the maternal ECG at the measurement site, $\operatorname{Md}_{k}(n)$ is a modulation function of the maternal ECG and can be caused from respiration and body movements, $F_{k}(n)$ is the FECG, $N_{k, \mathrm{EMG}}(n)$ is electromyographic (EMG) activity, $N_{k, 60 \mathrm{~Hz}}(n)$ is 50 or $60 \mathrm{~Hz}$ noise depending on the frequency of the power grid, $N_{k, \operatorname{amp}(n)}$ is amplifier electronic noise, $N_{k \text {,therm }}(n)$ is thermal noise from the signal source resistance, and $N_{k, \text { art }}(n)$ is artifact from patient and fetal movements, electrodes, and unknown sources $[2,4]$. If $S_{k}(n)$ is measured on the thoracic area or another position on the patient's periphery other than the anterior, lateral, or posterior abdominal wall, then $F_{k}(n)$ or the FECG is very small compared to the maternal ECG and is assumed zero. Furthermore, there is an increased probability of obtaining a noticeable FECG via an internal measuring device, such as an IC, placed in close proximity or touching the fetus versus the patient's periphery [2].

The signal can be written as

$$
S_{k}(n)=\operatorname{Md}_{k}(n) M_{k}(n)+F_{k}(n)+N_{k}(n),
$$

where

$$
\begin{aligned}
N_{k}(n)= & N_{k, \mathrm{EMG}}(n)+N_{k, 60 \mathrm{~Hz}}(n) \\
& +N_{k, \text { amp }}(n)+N_{k, \text { therm }}(n)+N_{k, \text { art }}(n) .
\end{aligned}
$$

The signal $S_{k}(n)$ bandwidth is usually from 0.05 to $100.0 \mathrm{~Hz}$. Finally, $M_{k}(n)$ and $F_{k}(n)$ overlap in frequency content, but the significant frequency content of $M_{k}(n)$ is from 0.05 to $40.0 \mathrm{~Hz}$ versus $F_{k}(n)$ which has its significant energy from 0.05 to $70.0 \mathrm{~Hz}[6]$.

\subsection{First reported IC method}

The first reported IC study focused on the design and placement of the catheter [2]. The paper presented an equivalent circuit model for the IC's interface to surrounding tissue and amniotic fluid.

The paper indicated that the IC was easy to insert during the early stages of delivery when the fetal head has not descended and engaged the pelvis. If the fetus had descended, extra resistance would be encountered during the insertion of the IC, which made placement and acquiring the FECG with a favorable SNR difficult.

The investigation did not perform signal processing. However, the paper did indicate that further processing via cancellation of the maternal ECG would be required to obtain a FECG with a SNR that HR and electrophysiological information could be readily determined. Finally, the analysis of the clinical data focused on the positioning of the IC and the resulting FECG amplitude [2].

\subsection{IC and adaptive filter method}

The adaptive filter technique for canceling the maternal ECG of the IC's ECG combines four adaptively filtered thoracic ECG signals and subtracts the resulting signals from a single IC's ECG [3]. The adaptive filter technique uses a conventional multiple channel least-mean-square (LMS) adaptive filter or noise canceler [7].

The reported IC and adaptive filter technique was developed for determining fetal HR information. The technique bandpass filters the analog thoracic and IC's ECG signals in a 15.0 to $40.0 \mathrm{~Hz}$ bandwidth before adaptive filtering is applied for maternal ECG cancellation [3]. Since the diagnostic bandwidth for an ECG is 0.05 to $100 \mathrm{~Hz}$, the FECG obtained using the reported technique cannot be used to determine electrophysiological information [8]. If electrophysiological information is desired, future research is necessary to determine whether a supplemental adaptive filter is necessary to eliminate baseline shifts that can occur from a diagnostic bandwidth of 0.05 to $100 \mathrm{~Hz}$ for an IC's ECG. If necessary, these baseline shifts may be eliminated by simple baseline removal methods that will not affect the spectrum of the signal. There may be some disadvantages of using a conventional adaptive filter that uses an autoregressive (AR) or autoregressive moving average (ARMA) adaptive section due to the larger order required to filter baseline shifts. Then an algorithm employing infinite impulse response (IIR) adaptive section should be utilized.

Furthermore, the reported IC and adaptive filter technique indicate that four leads are placed on the thoracic area to achieve maternal ECG cancellation. The large number of thoracic ECG leads is not user friendly and could create unnecessary confusion in a clinical environment. Future research is desirable to determine an alternate adaptive filter approach that requires only one thoracic ECG for maternal cancellation of an IC's ECG.

The clinical trials for the method consisted of acquiring patient data from 100 patients, of which 28 of the 100 had signal processing, performed to suppress the maternal ECG signal. The study reported that the 24 of the 28 patients or $86 \%$ had tracings with adequate quality that fetal HR information could be determined [3].

The reported results from the clinical trials of the adaptive filter technique are highly subjective and do not qualify their claims. They indicate that 24 out of 28 patient data sets that the maternal ECG was suppressed had adequate quality tracings that fetal HR information could be acquired. The article discusses some characteristics of the FECG of the IC's 
ECG, but there is no discussion of the effectiveness of the signal processing with various signal characteristics. In addition, the article does not qualify the adaptive filter's effectiveness with various signal characteristics to obtain a clinically useful FECG [3].

\subsection{Research contribution}

There have been several reports of maternal ECG cancellation via adaptive filtering and maternal ECG averaging and subtraction for FECG data obtained via the noninvasive abdominal wall ECG $[7,9,10,11,12,13,14,15]$. Presently, the adaptive filtering approach described above is the only reported maternal ECG cancellation technique for data obtained via the IC [3]. Since the IC's ECG is similar to the abdominal wall ECG signal, the use of maternal ECG averaging and subtraction on the IC's ECG is a natural progression and would fill the hole in the literature. The following research presents a real-time method for maternal ECG cancellation of an IC's ECG using one thoracic ECG and IC's ECG that is based on maternal ECG averaging and subtraction using modified maternal ECG complexes. In addition, the proposed method has been designed to function for a diagnostic bandwidth of 0.05 to $100.0 \mathrm{~Hz}$ and during the occurrence of baseline shifts.

Since previous methods for acquiring a FECG via an IC's ECG did not perform maternal ECG cancellation and/or presented little to no analysis of their maternal ECG cancellation method, the main contribution of this research is an original effectiveness analysis for the proposed method. This paper includes several detailed subjective and objective analyses with synthesized and clinical data. Furthermore, the analysis will examine the clinical usefulness of the resulting IC's ECG found with the proposed method.

\section{METHOD}

This section presents a real-time digital signal processing (DSP) method for canceling the maternal ECG of the IC's ECG to fulfill the first goal of Section 1. However, acquiring a clinically useful FECG via an IC requires maternal ECG cancellation along with additional analog and DSP. The analog processing includes a custom ultralow noise preamplifier and bandpass filter. The additional DSP performs FECG SNR enhancement after the maternal ECG has been canceled. Figure 1 presents a detailed block diagram of the system. Since the focus of this paper is on the real-time technique for maternal ECG cancellation, details of the analog signal processing and FECG SNR enhancement via FECG averaging will not be discussed. A detailed discussion of the analog signal processing is used to acquire the clinical data of this study, and the DSP for FECG averaging of this study have been previously published $[5,16]$.

\subsection{Data acquisition}

Following the analog signal processing, the IC's and thoracic ECGs are digitized with a sampling rate of $1 \mathrm{kHz}$ and 16 bits of quantization. Using notation from Section 2.1, the

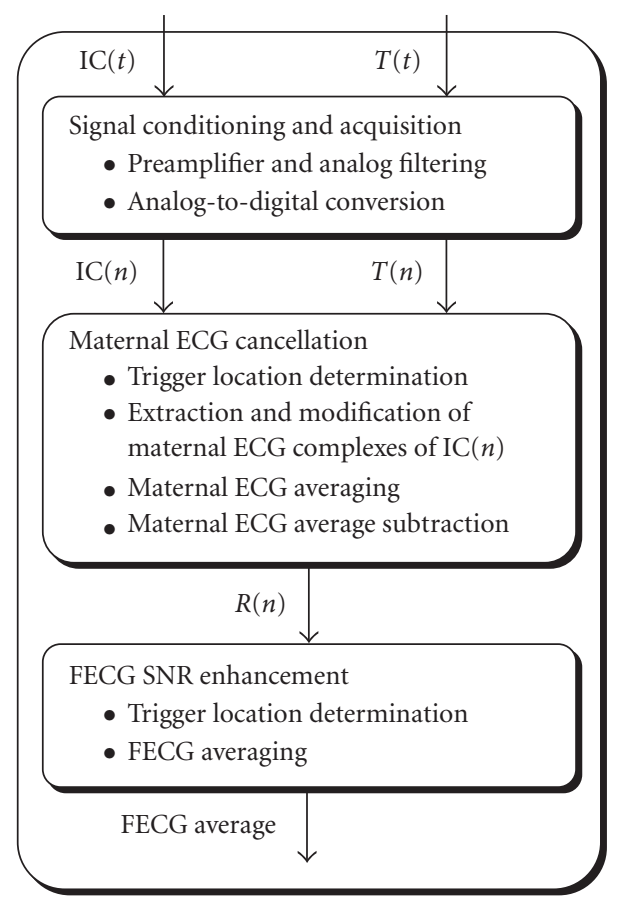

FIGURE 1: Block diagram of the signal conditioning, data acquisition, and DSP performed by the system.

digitized IC’s ECG can be written as

$$
\mathrm{IC}(n)=S_{0}(n)
$$

The digitized thoracic ECG can be written as

$$
T(n)=S_{1}(n),
$$

where the FECG component of $T(n)$ is negligible, as discussed in Section 2.1, and the noise from various sources is also negligible compared to the IC's ECG.

During data acquisition, the thoracic and IC's ECGs are digitized into blocks with $N^{\prime}$ samples. The total number of samples in each block is generally set to 8000 samples or 8.0 seconds of data for a $1 \mathrm{kHz}$ sampling rate. Setting $N^{\prime}$ to 8000 samples creates a window that will produce an acceptable delay at the start of the data acquisition and capture an adequate number of maternal ECG complexes for the cancellation algorithm to function properly.

Figure 2 presents the first and second eight seconds blocks of a clinical data set to demonstrate the method. Figures $2 \mathrm{a}$ and $2 \mathrm{~b}$ are the thoracic and IC's ECGs, respectively, from 0.0 to 8.0 seconds. Figures $2 \mathrm{c}$ and $2 \mathrm{~b}$ are the thoracic and IC's ECG, respectively, from 8.0 to 16.0 seconds. The display of the thoracic and IC's ECGs of Figures $2 a$ and 2b, respectively, occur 8.0 seconds after the start of the program. Therefore, time equal to zero on the plots of Figures $2 \mathrm{a}$ and $2 \mathrm{~b}$ correspond to eight seconds after the start of the data acquisition and program. 


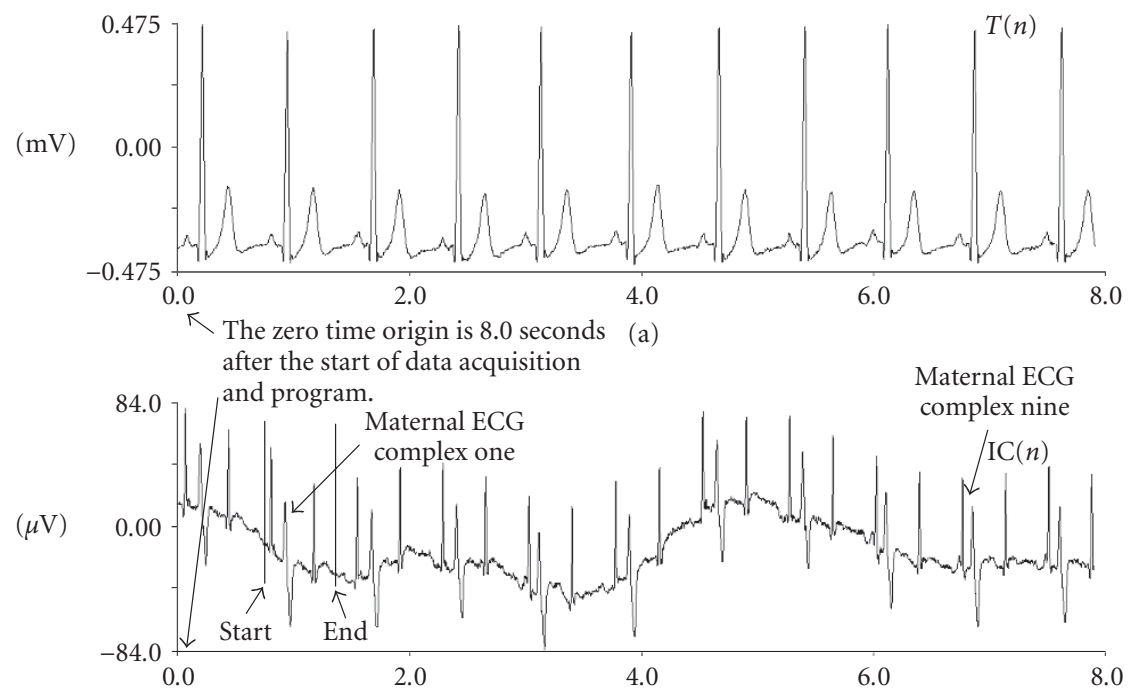

(b)

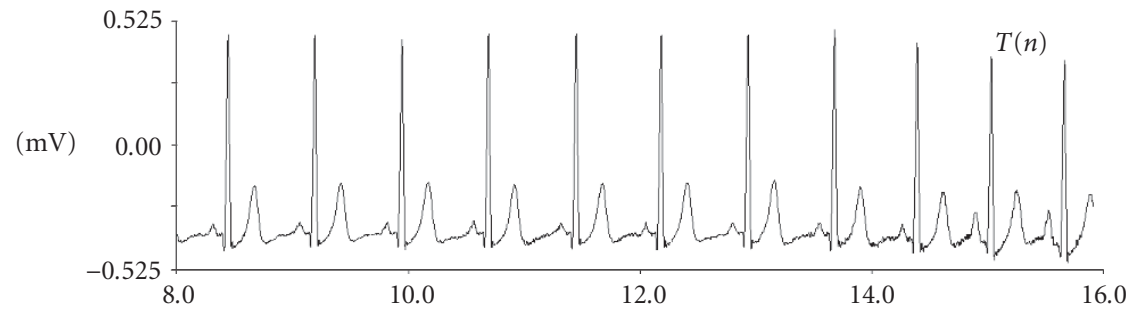

(c)

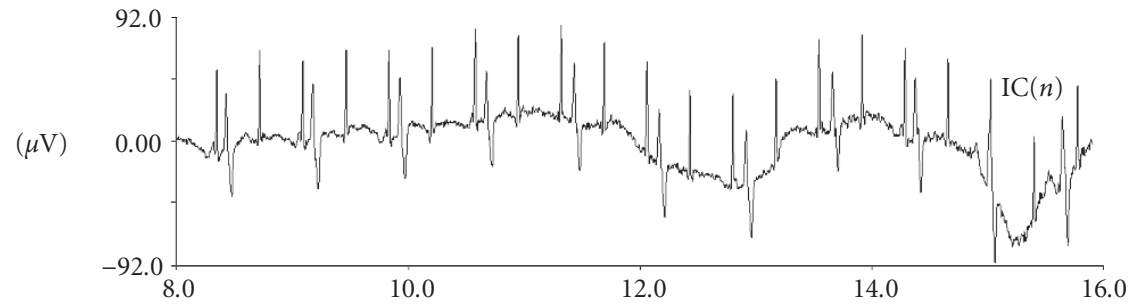

(d)

FIGURE 2: First and second eight seconds blocks of continuous IC clinical data used to illustrate the method (all $x$-axes in seconds). (a) Thoracic ECG from 0.0 to 8.0 seconds. (b) IC's ECG from 0.0 to 8.0 seconds. (c) Thoracic ECG from 8.0 to 16.0 seconds. (d) IC's ECG from 8.0 to 16.0 seconds.

\subsection{Maternal ECG cancellation}

The proposed technique achieves maternal ECG cancellation via maternal ECG averaging and subtraction for an IC's ECG. A thoracic ECG is utilized as a trigger for extracting and aligning the maternal ECG complexes from the IC's ECG to form the maternal ECG average. The extracted maternal ECG complexes are modified, before averaging, to reduce FECG QRS complex residual in the maternal ECG average used for subtraction.

The following describes the method where Figures 1 through 7 are used to facilitate the explanation. The 0.5 to 2.0 seconds of the thoracic and IC's ECGs of Figures $2 \mathrm{a}$ and $2 \mathrm{~b}$, respectively, are used to demonstrate the method in Figure 3. The first two seconds of the thoracic and IC's ECGs of Figures $2 \mathrm{a}$ and $2 \mathrm{~b}$, respectively, are used to demonstrate the method in Figures 4 and 6. The subsections of the eight seconds block of data were used to clearly illustrate the method graphically.

The start and end of an example maternal ECG complex of an IC's ECG is indicated in Figures $2 \mathrm{~b}$ and $4 \mathrm{~b}(\mathrm{i})$, and Figure $4 \mathrm{a}(\mathrm{i})$ labels the $\mathrm{P}, \mathrm{QRS}$, and T waves for a maternal ECG complex of the thoracic ECG. The P wave is the atrium depolarization, QRS waves in the ventricular depolarization, and the $\mathrm{T}$ wave is the ventricular repolarization.

\subsubsection{Trigger location determination}

The first step in Figure 1 for maternal ECG cancellation determines the trigger locations for maternal ECG averaging and subtraction. To avoid inaccurate trigger locations from low- and high-frequency noise via peak detection, the thoracic ECG $T(n)$ is bandpass filtered from 2.0 to $35.0 \mathrm{~Hz}$ to 


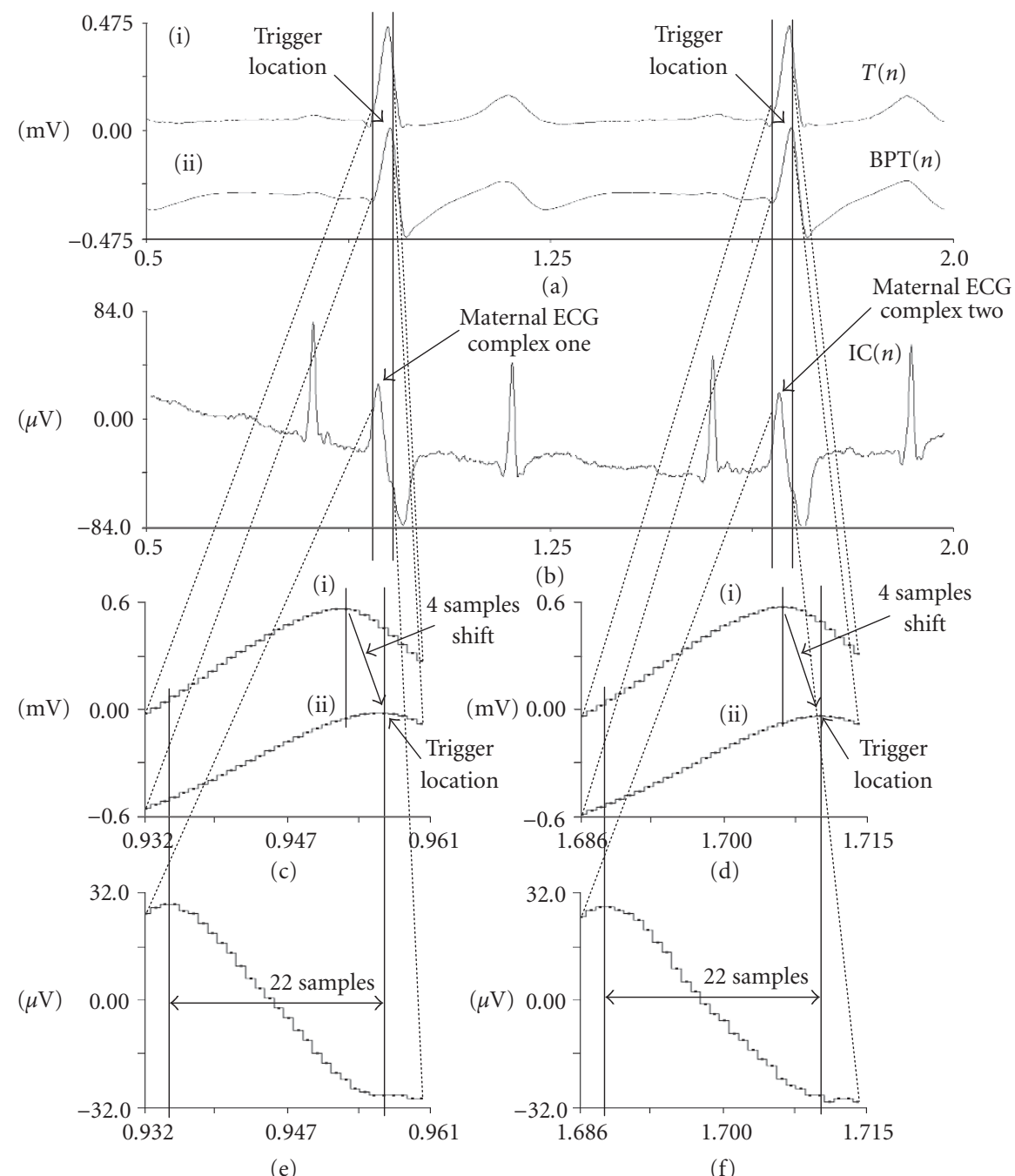

FIgURE 3: Demonstration of the thoracic ECG bandpass filter shift and compensation along with trigger location determination (all $x$ axes in seconds). (a)(i) $T(n)$. (a)(ii) BPT(n). (b) IC's ECG. (c)(i) and (ii) A portion of maternal ECG complex one of the $T(n)$ and BPT( $n)$, respectively. (d) (i) and (ii) Same as (c) but for maternal ECG complex two. (e) and (f) A portion of the IC's ECG for maternal ECG complexes one and two, respectively.

form $\operatorname{BPT}(n)$. A second-order IIR Butterworth filter was utilized. Figures $3 \mathrm{a}(\mathrm{i})$ and $4 \mathrm{a}(\mathrm{i})$ are the thoracic ECG $T(n)$ and Figures $3 \mathrm{a}(\mathrm{ii})$ and $4 \mathrm{a}(\mathrm{ii})$ are the bandpass filtered thoracic ECG BPT( $n)$. Figures 3c(i), 3d(i), 3c(ii), and 3d(ii) present a detailed view of the thoracic ECG of Figure $3 a(i)$ and the filtered thoracic ECG of Figure 3a(ii), respectively, in sample form. Figures $3 \mathrm{a}, 3 \mathrm{c}$, and $3 \mathrm{~d}$ demonstrate the shift that results from the bandpass filter where the four-sample shift from filtering is indicated on the figures.

Next, the method determines the maximum of the filtered thoracic ECG BPT $(n)$. Fifty percent of the maximum is found and used by the peak detection algorithm as a threshold voltage for detecting the maternal ECG's R wave peaks or trigger locations of $\mathrm{BPT}(n)$. The detected trigger locations are indicated on Figures $3 \mathrm{a}(\mathrm{ii}), 3 \mathrm{c}(\mathrm{ii})$, and $3 \mathrm{~d}$ (ii) and by the circles on the $x$-axis of Figures $4 \mathrm{a}$ and $4 \mathrm{~b}$.

\subsubsection{Extraction and modification of maternal ECG complexes}

The second step extracts and then modifies each maternal ECG complex of the IC $(n)$. The modification consists of replacing the FECG's QRS complexes of the extracted maternal ECG complexes with a linear interpolation.

The maternal ECG complexes are extracted from the IC $(n)$ via the trigger locations. The start of each maternal ECG complex of IC $(n)$ is $M 1$ samples before each trigger location where $M 1$ is $20 \%$ of the sampling rate. The end of each maternal ECG complex is $M 2$ minus $M 1$ samples after the trigger location where $M 2$ is $60 \%$ of the sampling rate. Each maternal ECG complex is extracted by acquiring $M 2$ samples of IC $(n)$ starting at each trigger location minus $M 1$ samples. The four-sample shift from the bandpass filter to determine the trigger locations has been added into $M 1$ to 


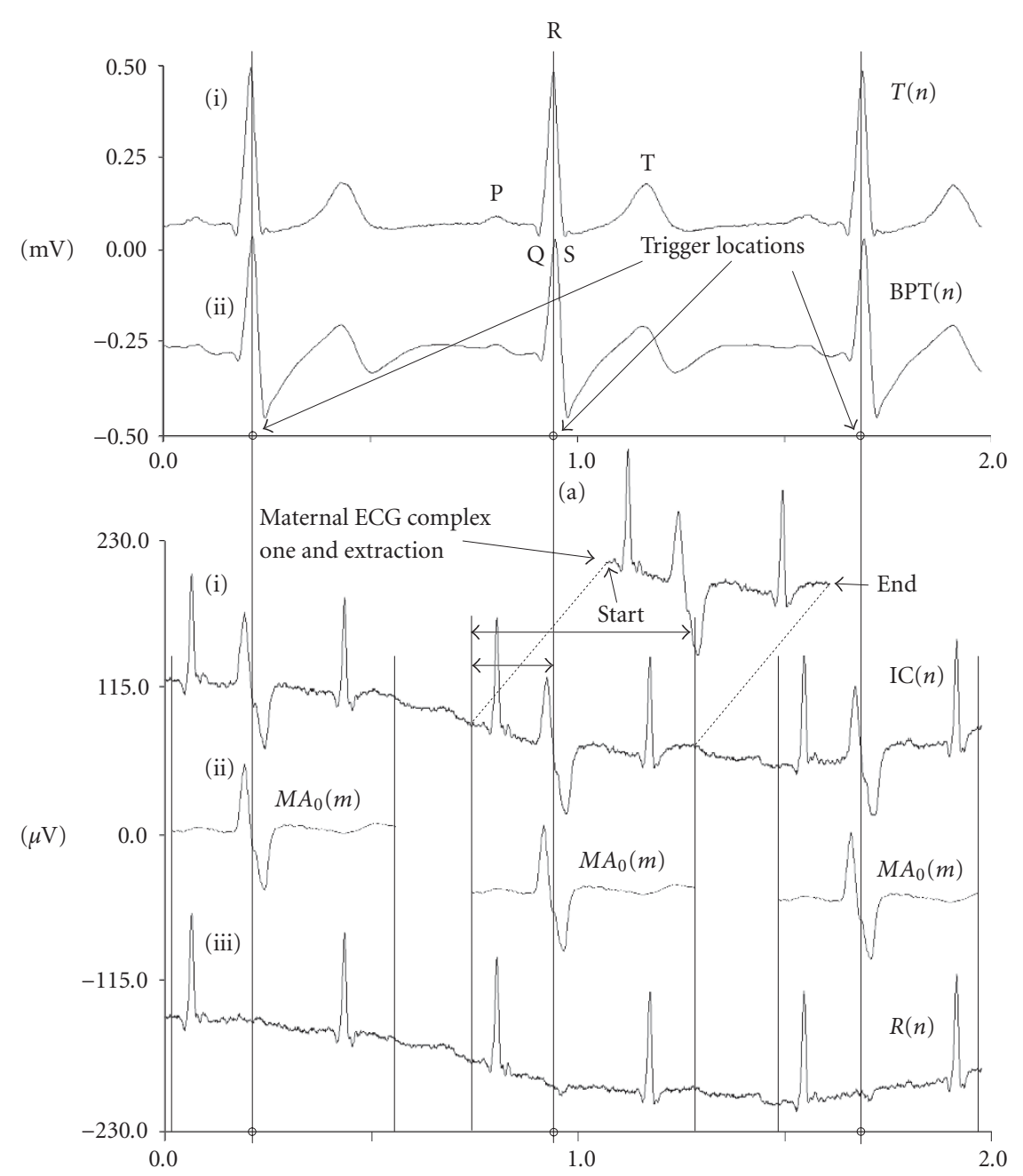

(b)

FIGURE 4: Demonstration of the maternal ECG cancellation algorithm using a maternal ECG average calculated via modified maternal ECG complexes (all $x$-axes in seconds). (a)(i) Thoracic ECG. (a)(ii) Bandpass filtered thoracic ECG. (b)(i) IC's ECG. (b)(ii) Maternal ECG average aligned for subtraction. (b)(iii) IC's ECG with the maternal ECG canceled.

compensate for the shift. Maternal ECG complexes that occur at the beginning and end of the $i$ th block of data are not extracted since they can contain partial complexes from the start and termination of the block, respectively. The value of $20 \%$ of the sampling rate for $M 1$ was fixed for the eight clinical data sets studied. However, the value of $60 \%$ of the sampling rate for $M 2$ varied from $60 \%$ to $90 \%$ for the eight clinical data sets studied, where seven of the data sets had values of $60 \%$ for $M 2$ and one data set had a value of $90 \%$ for $M 2$.

Figure $4 \mathrm{~b}(\mathrm{i})$ indicates graphically the values $M 1$ and $M 2$ in relation to the second circled trigger location of Figure 4a for extracting the maternal ECG complexes IC $(n)$. Figure 5a presents the extracted maternal ECG complexes from the first eight second block of data of the IC's ECG of Figure 2b where maternal ECG complex one and nine are labeled on Figures $2 \mathrm{~b}$ and $5 \mathrm{a}$. The second maternal ECG complex on Figure $2 b$ is labeled maternal ECG complex one since the first maternal ECG complex of each 8.0 seconds block of data may only be a partial complex and cannot be used for averaging. The last maternal ECG complex of the 8.0 seconds block of Figure $2 \mathrm{~b}$ is not used for the same reason.

Figure 3 demonstrates that the trigger locations determined from the bandpass filtered thoracic ECG correspond to the same morphological locations for each maternal ECG complexes of the IC's ECG. Therefore, the trigger locations from the bandpass filtered thoracic ECG can be used for extracting, averaging, and subtracting the maternal ECG complexes of the IC's ECG. Figures $3 \mathrm{a}$ and $3 \mathrm{~b}$ present the thoracic ECG and IC's ECG, respectively, along with maternal ECG complexes one and two to be extracted from the IC's ECG. Figures $3 \mathrm{c}$ and $3 \mathrm{~d}$ present a detailed view of a portion of the maternal ECG complexes from the thoracic ECG and bandpass filtered thoracic ECG of Figure 3a. Figures $3 \mathrm{e}$ and $3 \mathrm{f}$ present a detailed view of a portion of the maternal ECG complexes from the IC's ECG 


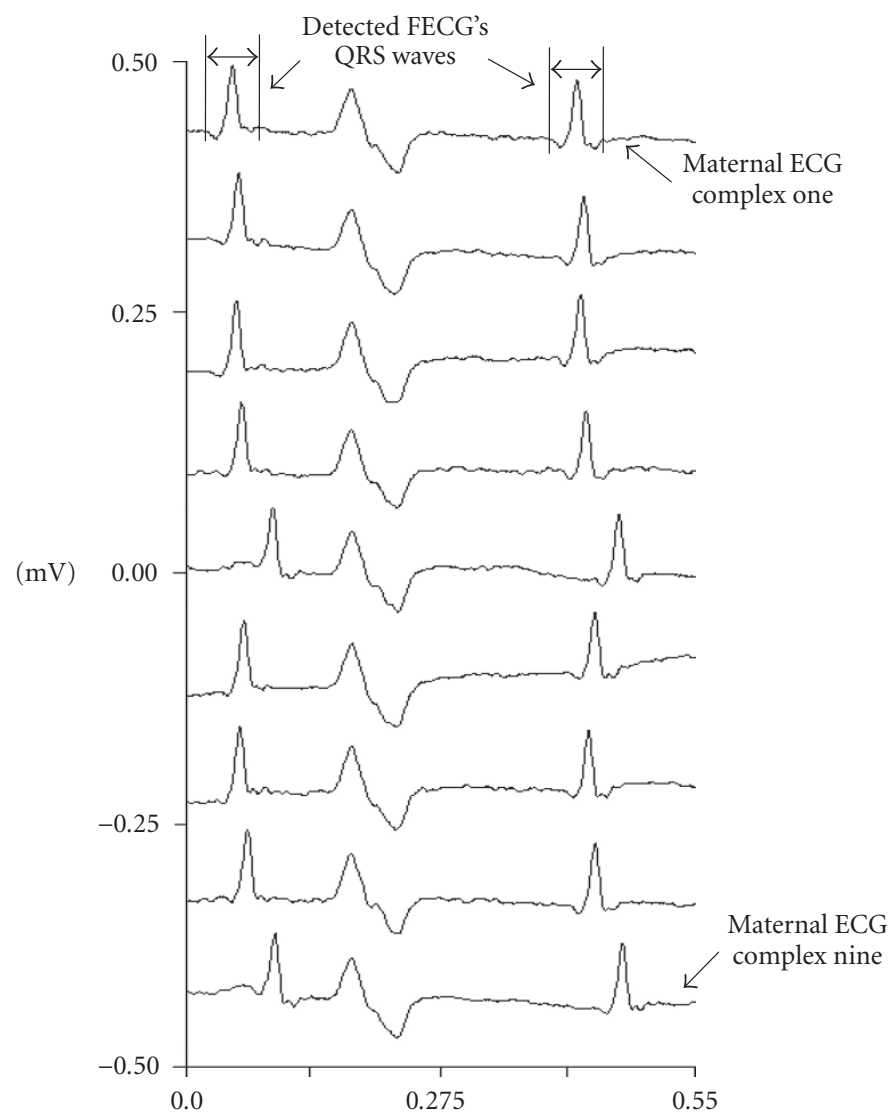

(a)

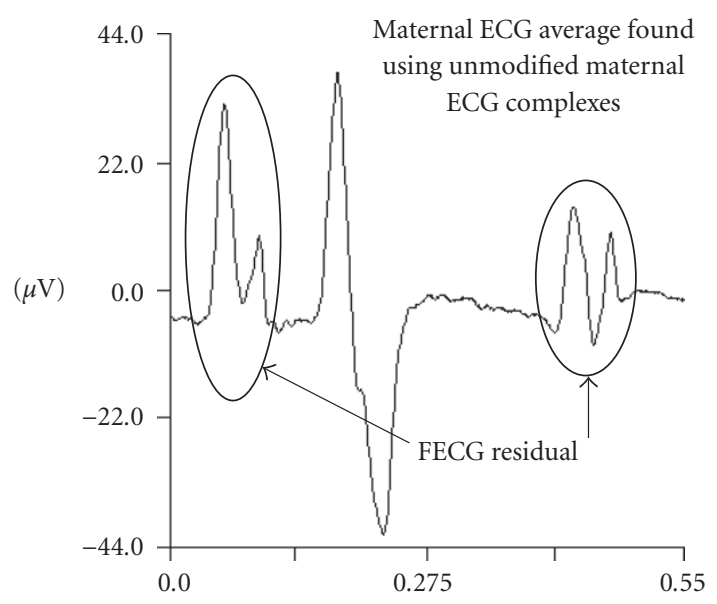

(c)

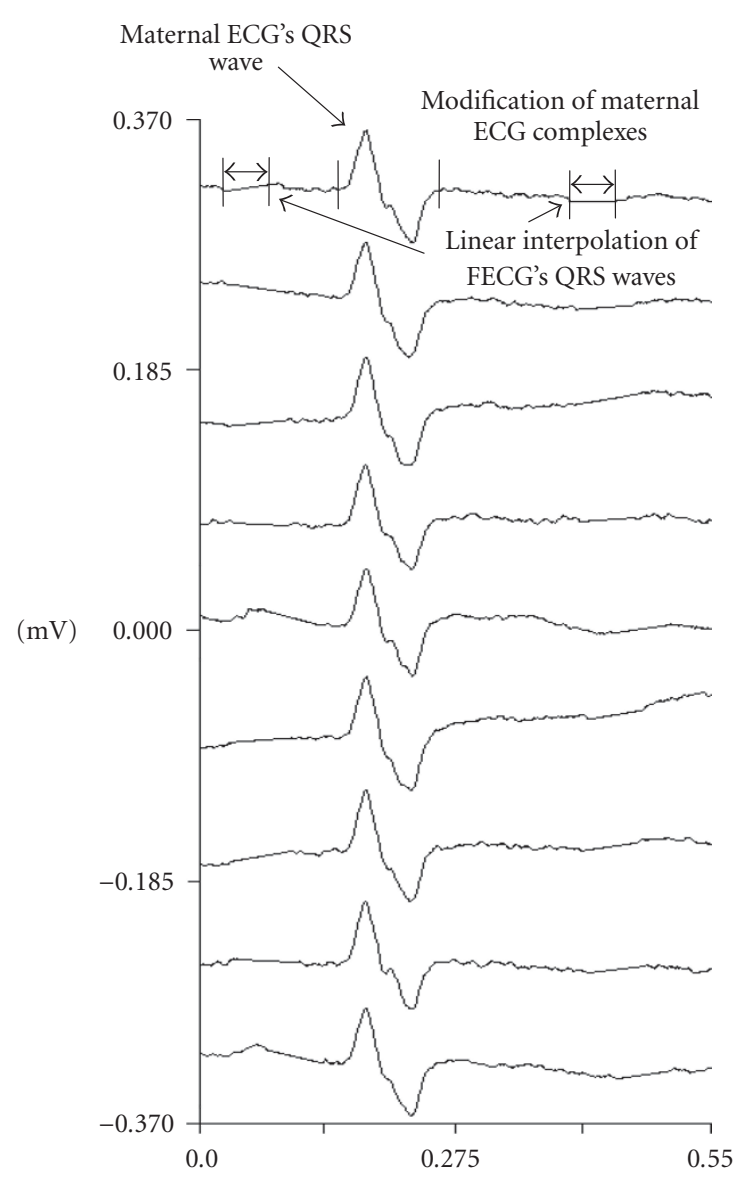

(b)

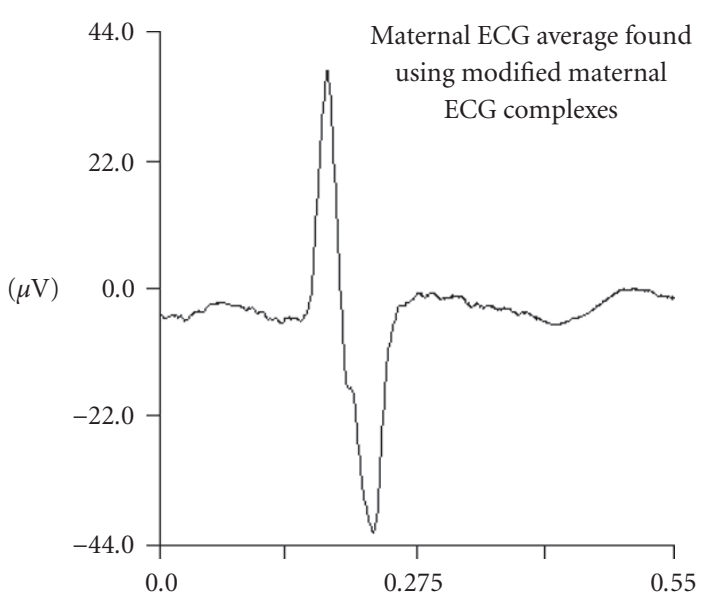

(d)

FIGURE 5: Comparison of unmodified versus modified maternal ECG complexes to calculate the maternal ECG average (all $x$-axes in seconds). (a) Unmodified maternal ECG complexes. (b) Modified maternal ECG complexes. (c) Maternal ECG average found using unmodified maternal ECG complexes of (a). (d) Maternal ECG average found using modified maternal ECG complexes of (b).

of Figure $3 \mathrm{~b}$. From the trigger locations indicated on Figures $3 \mathrm{a}(\mathrm{ii}), 3 \mathrm{c}(\mathrm{ii})$ and $3 \mathrm{~d}(\mathrm{ii})$, the peaks of the IC's maternal ECG complexes are 22 samples before the trigger locations for maternal ECG complex one and two presented in Figure 3. Table 1 presents the bandpass filter shift and number of samples before the trigger locations to the peaks of the IC's maternal ECG complexes for the clinical data of Figures $2 \mathrm{a}$ and $2 \mathrm{~b}$. Since the peaks of the IC's maternal ECG 
TABLE 1: Bandpass filter shift and trigger location verification for clinical data of Figures $2 \mathrm{a}$ and $2 \mathrm{~b}$.

\begin{tabular}{lccccccccc}
\hline Maternal ECG complex \# & One & Two & Three & Four & Five & Six & Seven & Eight & Nine \\
\hline $\begin{array}{l}\text { Bandpass filter } \\
\text { Shift (samples) }\end{array}$ & 4 & 4 & 5 & 4 & 4 & 4 & 4 & 4 & 4 \\
$\begin{array}{l}\text { IC's ECG trigger location } \\
\text { Verification (samples) }\end{array}$ & 22 & 22 & 23 & 22 & 22 & 22 & 23 & 22 & 23 \\
\hline
\end{tabular}

complexes occur consistently 22 to 23 samples before the trigger locations, the trigger locations accurately determine the same morphological location for each IC's maternal ECG complex for this data set. Similar results have been observed for other data sets.

The detection of each FECG's QRS wave is performed similarly to the detection of the maternal ECG complexes of the IC $(n)$. The maternal ECG complexes are bandpass filtered from 2.0 to $35.0 \mathrm{~Hz}$ to avoid inaccurately detecting of the FECG QRS waves from low- and high-frequency noise. A threshold voltage for peak detection of each maternal ECG complex is found by determining $50 \%$ of the maximum value for each filtered maternal ECG complex. The peak detection algorithm from the analysis library is used to find the FECG's QRS wave peaks for each maternal ECG complex. The peak detection algorithm ignores the FECG's QRS wave(s) that occurs during the maternal ECG's QRS wave. Figure 5a indicates the detected FECG QRS waves for the extracted maternal ECG complex one.

For each detected FECG's QRS wave peak outside of the maternal ECG's QRS wave, a linear interpolation is performed between the first and last samples of the detected waves. The width of the FECG's QRS wave for the linear interpolation is set to $M 3$ samples or $2 \%$ of the sampling rate. The replacement of the FECG's QRS wave with the linear interpolation significantly reduces the signal energy of the FECG in each maternal ECG complex. Figures 5a and $5 \mathrm{~b}$ demonstrates the linear interpolation used to replace the FECG QRS's wave(s) of each maternal ECG complex. Maternal ECG complex one of Figure 5a indicates the location, labeled with double arrows and duration, $M 3$, of the linear interpolation that will be performed in place of the two present FECG's QRS complexes. Figure 5b presents the extracted maternal ECG complexes where the FECG's QRS waves have been replaced by the linear interpolation. The portions of maternal ECG complex one that the interpolation has been performed are indicated in Figure 5b.

\subsubsection{Maternal ECG averaging}

Step three determines the maternal ECG average from the modified maternal ECG complexes. The maternal ECG average is accumulative from 0 through $i$ blocks of data. The average is calculated for each $i$ block of data and averaged with the previous average found for $i-1$ block of data. Maternal ECG complexes that occur at the beginning and end of the $i$ th block of data are not included in averaging since they can contain partial complexes from the start and termination of the block. Since the maternal ECG average can have a DC offset, a DC offset adjust has been incorporated in the algorithm after updating the average for each $i$ th block of data. The offset of the maternal ECG average is calculated by averaging $M 4$ samples from the beginning and end of the maternal ECG average. Five percent of the sampling rate has been determined experimentally to be a good number for M4. Then, the offset is subtracted from the maternal ECG average.

The method is designed to detect the FECG's QRS complexes with a high SNR and replace with a linear interpolation since these complexes can produce a significant residual during maternal ECG averaging. A FECG with a high SNR would be a FECG's QRS complex that is greater than half the amplitude of the maternal ECG's QRS complex.

Figures $5 \mathrm{c}$ and $5 \mathrm{~d}$ present the maternal ECG average found via averaging the unmodified and modified maternal ECG complexes of Figures $5 a$ and $5 b$, respectively. The maternal ECG average found via the unmodified maternal ECG complexes of Figure 5a was presented as a comparison with the maternal ECG average calculated from the modified complexes of Figure $5 \mathrm{~b}$ to demonstrate the need for linear interpolation for a FECG with a high SNR. The average found via the modified complexes clearly has less FECG residual. The resulting FECG residual from using unmodified maternal ECG complexes for a FECG with a high SNR during averaging is indicated in Figure 5c.

\subsubsection{Maternal ECG average subtraction}

The fourth step subtracts the maternal ECG average from each maternal ECG complex of the IC's ECG IC $(n)$. The maternal ECG average is aligned with each maternal ECG complex using the trigger locations used to find the average.

Figure $4 \mathrm{~b}$ (ii) presents the maternal ECG average aligned for subtraction from the IC's ECG IC $(n)$ of Figure $4 \mathrm{~b}(\mathrm{i})$ via the trigger locations indicated by the circles on the $x$-axis of Figures $4 \mathrm{a}$ and $4 \mathrm{~b}$. The resulting IC's ECG $R(n)$ is presented in Figure 4b(iii).

Figure 6 demonstrates the same clinical data as Figure 4. However, the maternal ECG average used for subtraction was formed from the unmodified maternal ECG complexes. The result from applying the maternal ECG average from the unmodified complexes was presented to demonstrate the effectiveness of modifying the maternal ECG complexes. Figures $6 \mathrm{a}(\mathrm{i})$ and $6 \mathrm{a}(\mathrm{ii})$ present the thoracic and bandpass filter thoracic ECGs. Figure 6b(ii) presents the maternal ECG average aligned for subtraction from the IC's ECG IC $(n)$ of Figure $6 \mathrm{~b}(\mathrm{i})$ via the trigger locations indicated by the circles on the $x$-axis of Figures $6 \mathrm{a}$ and $6 \mathrm{~b}$. The resulting IC's ECG 


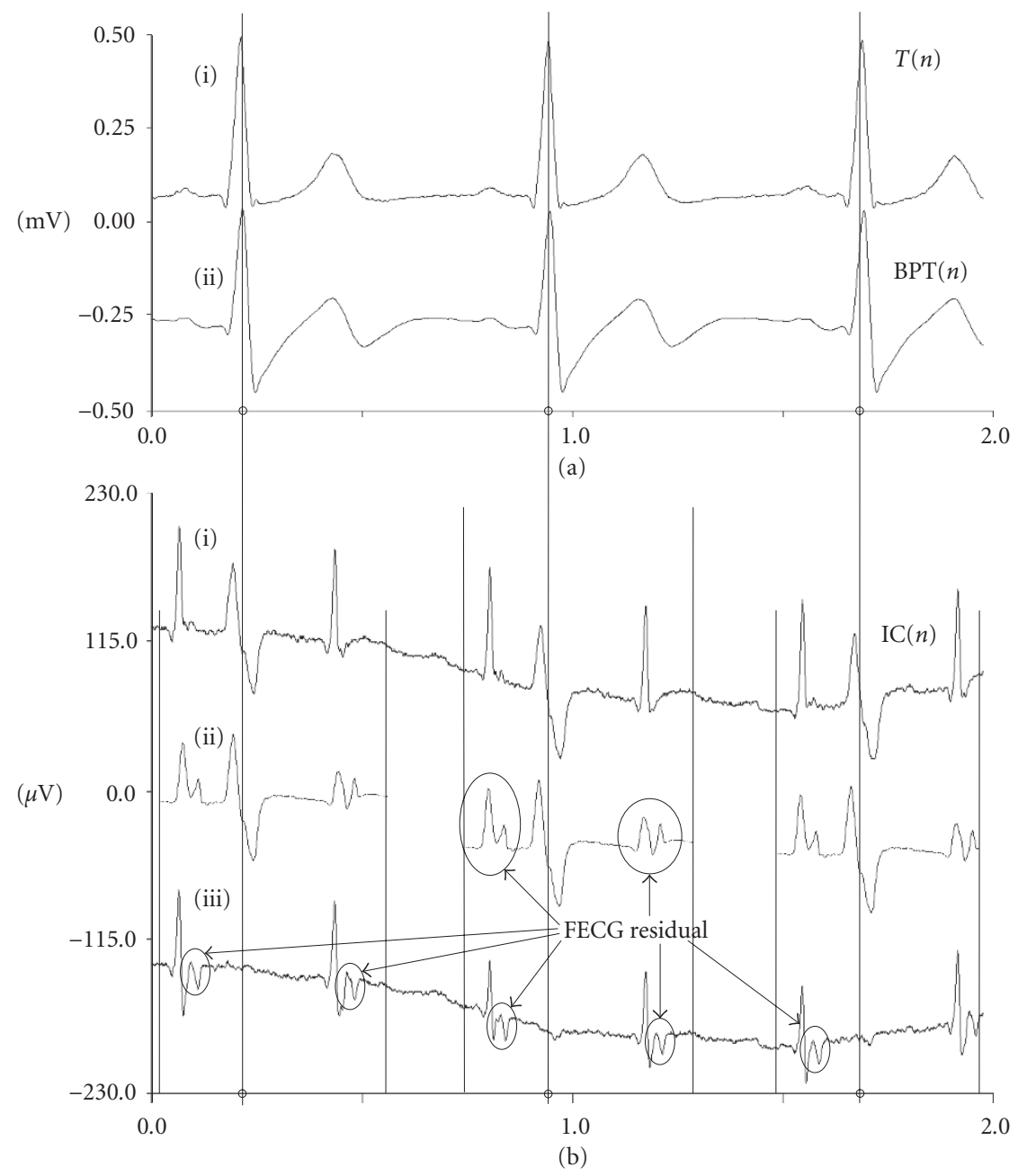

FIGURE 6: Demonstration of the maternal ECG cancellation algorithm using a maternal ECG average calculated via unmodified maternal ECG complexes (all $x$-axes in seconds). (a)(i) Thoracic ECG. (a)(ii) Bandpass filtered thoracic ECG. (b)(i) IC's ECG. (b)(ii) Maternal ECG averages aligned for subtraction. (b)(iii) IC's ECG with the maternal ECG canceled.

$R(n)$ is presented in Figure $6 \mathrm{~b}$ (iii). The large circles on Figures $6 \mathrm{~b}$ (ii) and $6 \mathrm{~b}$ (iii) indicate the significant FECG residual that results compared to Figures $4 \mathrm{~b}$ (ii) and $4 \mathrm{~b}$ (iii).

The maternal ECG cancellation method block size can be varied from 8.0 to 10.0 seconds. Figure 7 presents clinical data for a block size of 10,000 samples or 10.0 seconds instead of 8.0 seconds. Figures $7 \mathrm{a}(\mathrm{i})$ and $7 \mathrm{a}(\mathrm{ii})$ are the thoracic and bandpass filtered thoracic ECGs, respectively, from 0.0 to 10.0 seconds, and Figures $7 \mathrm{~b}(\mathrm{i})$ and $7 \mathrm{~b}$ (ii) are the IC's ECG and IC's ECG with the maternal ECG canceled, respectively, from 0.0 to 10.0 seconds. Figures $7 \mathrm{c}(\mathrm{i})$ and $7 \mathrm{c}$ (ii) are the thoracic and bandpass filtered thoracic ECGs, respectively, from 10.0 to 20.0 seconds, and Figures $7 \mathrm{~d}$ (i) and $7 \mathrm{~d}$ (ii) are the IC's ECG and IC's ECG with the maternal ECG canceled, respectively, from 10.0 to 20.0 seconds. There is a 10.0 seconds delay from the start of the data acquisition and program to the display of the signals. The block size can be changed to a larger value. However, the delay time from the start of acquisition and program to the display of the processed signals will increase.

\section{RESULT}

The objective of this section is to ascertain the effectiveness of the proposed method for maternal ECG cancellation with synthesized and clinical data to fulfill the second goal of Section 1. The focus of this section will be on subjective and objective analyses of the maternal ECG cancellation method on the IC's ECG. In addition, the analyses presented will be rigorous and designed to thoroughly probe the strengths and weaknesses of the technique.

The Results section analyzes synthesized and clinical data from eight patients. The clinical data sets presented are intended to be representative data. An IC's ECG will typically have noise, nonideal signal components from a variety of sources, and strong to weak or indistinguishable FECG 


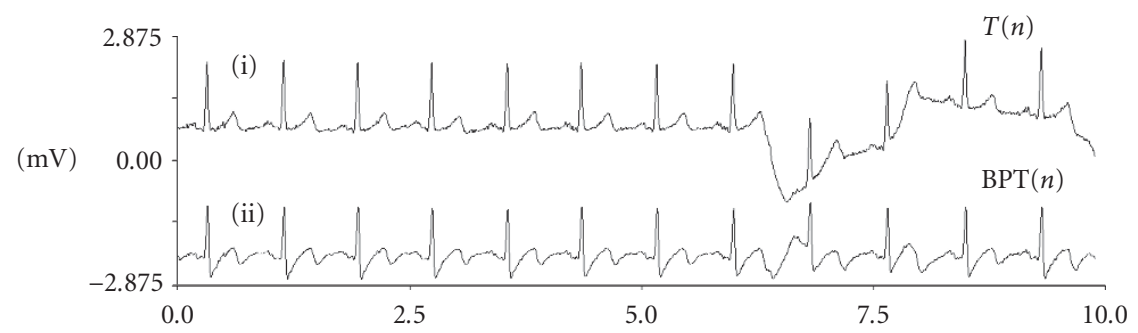

(a)

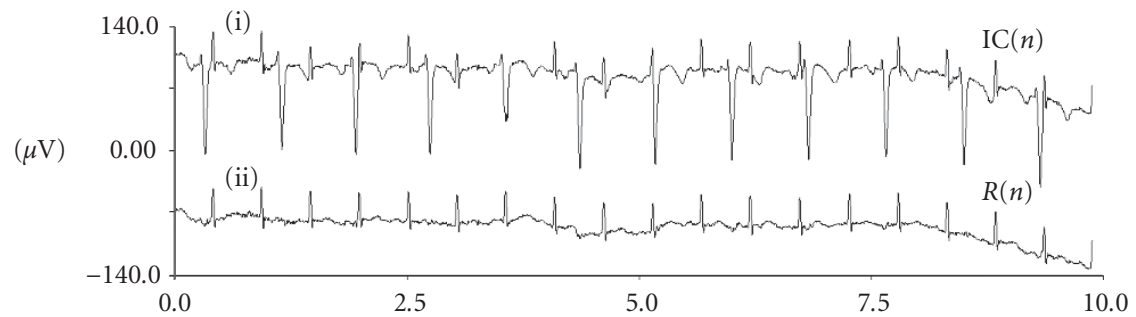

(b)

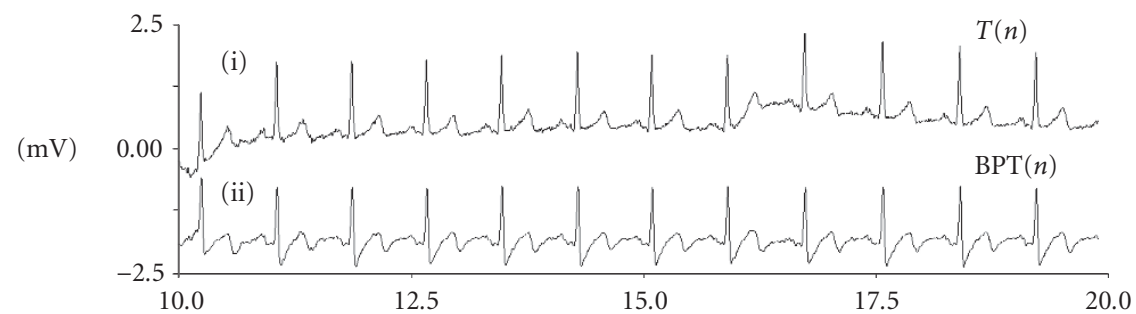

(c)

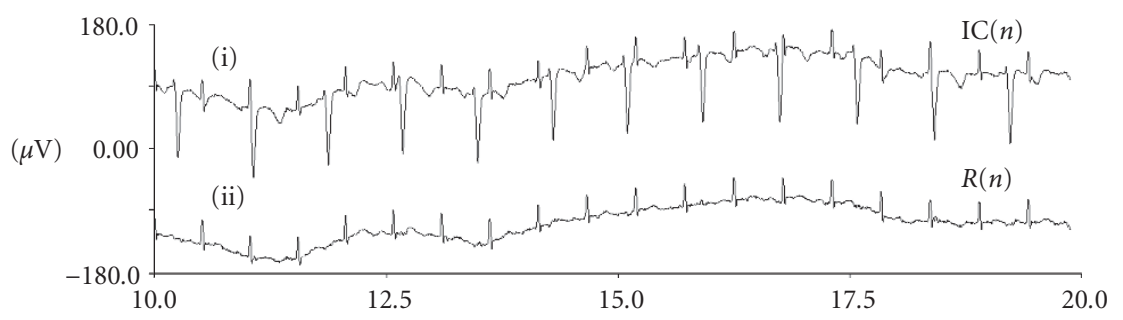

(d)

Figure 7: First and second ten seconds blocks of continuous IC clinical data (all $x$-axes in seconds). (a)(i) Thoracic ECG from 0.0 to 10.0 seconds. (a)(ii) Bandpass filtered thoracic ECG from 0.0 to 10.0 seconds. (b)(i) IC's ECG from 0.0 to 10.0 seconds. (b)(ii) IC's ECG with the maternal ECG canceled from 0.0 to 10.0 seconds. (c)(i) Thoracic ECG from 10.0 to 20.0 seconds. (c)(ii) Bandpass filtered thoracic ECG from 10.0 to 20.0 seconds. (d)(i) IC's ECG from 10.0 to 20.0 seconds. (d)(ii) IC's ECG with the maternal ECG canceled from 10.0 to 20.0 seconds.

compared to other signals of the IC's ECG. Therefore, not all the data sets presented are the most ideal, but samples that contain ideal, typical, and nonideal data. By using representative data and various test conditions and measures in the analysis of the method, the reader should gain much insight into the effectiveness of the technique. Finally, the clinical data of this study was obtained from patients at the University of Tennessee Medical Center Knoxville using a protocol approved by the institutional review board (IRB).

\subsection{Synthesized data}

Synthesized data was used to verify the effectiveness and accuracy of the proposed method for maternal ECG cancellation via a subjective visual inspection of the data and six objective numerical measures. Synthesized data as opposed to clinical data was initially applied to test the method because a pure FECG signal of clinical data is unknown. Therefore, an objective comparison of the resulting FECG with a pure FECG is not possible. With synthesized data, the pure FECG 


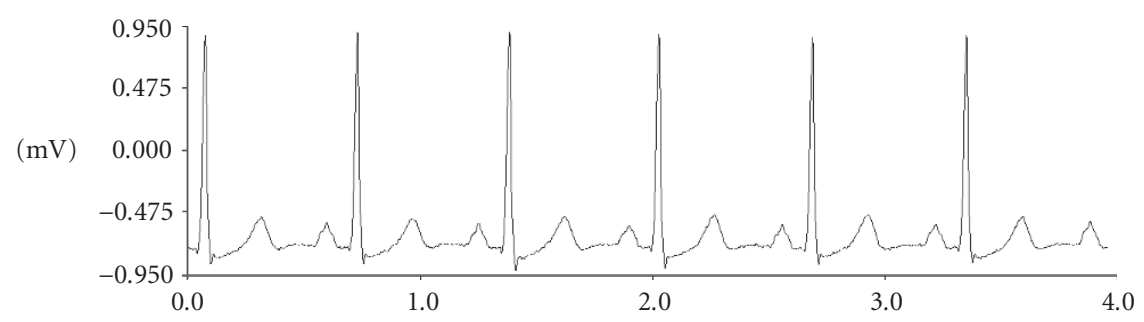

(a)

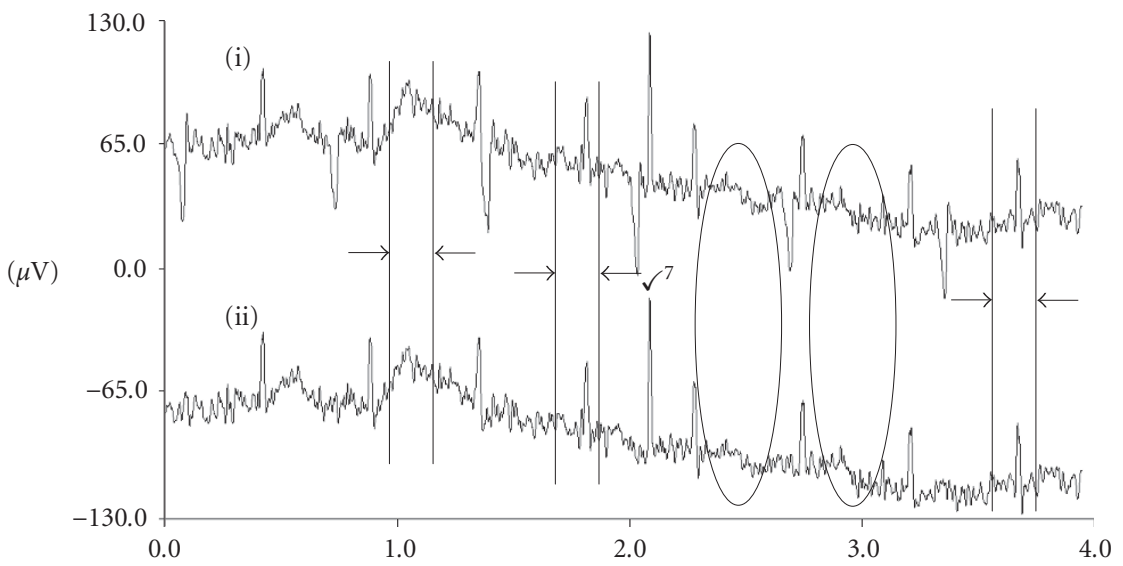

(b)

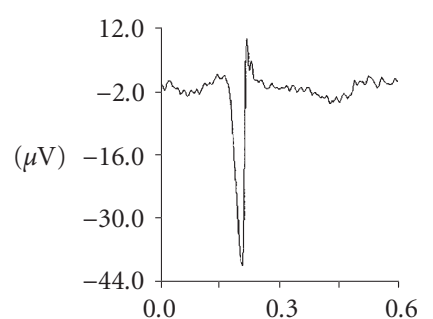

(c)

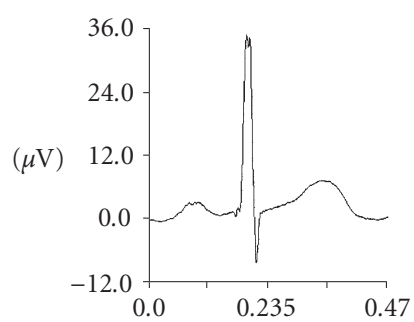

(d)

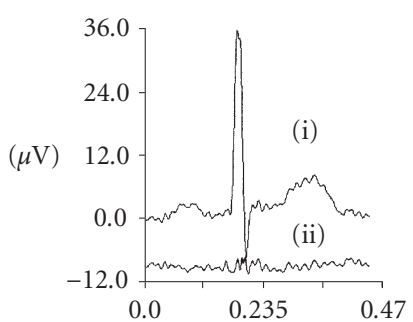

(e)

Figure 8: Intrauterine catheter synthesized data set 1 (all $x$-axes in seconds). (a) Thoracic ECG. (b)(i) Intrauterine catheter signal. (b)(ii) Intrauterine catheter signal with the maternal ECG canceled. (c) Maternal ECG average of the intrauterine catheter signal. (d) Ideal FECG complex of the signal of (b)(ii). (e)(i) FECG average from the signal of (b)(ii) (47 complexes averaged). (e)(ii) Resulting signal from subtracting FECG signal in (d) from (e)(i).

signal is known and an objective analysis can be performed between the pure and resulting FECG signals. Finally, a second set of synthesized data was employed to simulate a signal characteristic that evoked failure of the proposed method to demonstrate a vulnerability of the algorithm.

Clinical data from two patients of the IC study were combined to create realistic synthesized data. Figure $8 \mathrm{a}$ is the thoracic ECG from patient 5 of the IC study. Figure $8 b(i)$ is an IC's ECG from patient 5 of the IC study that did not contain a FECG. A pure FECG strip was synthesized from an averaged FECG complex from patient 3 of the IC study and added to the IC's ECG of Figure $8 \mathrm{~b}(\mathrm{i})$. The data of Figure 9 is synthesized from patient 1 data of Figure 10 via modulating the IC's ECG of Figure $10 b(i)$.

\subsubsection{Subjective analysis}

Figure 8 demonstrates the two steps of canceling the maternal ECG and improving the FECG's SNR. Figure 8b(ii) presents the resulting IC's ECG $R(n)$ from applying the proposed maternal ECG cancellation algorithm. Only four of the eight seconds block of data is presented in Figure 8 to clearly illustrate the signal processing. Figure $8 \mathrm{c}$ is the maternal ECG average used in the suppression. A subjective analysis or visual inspection can see that the maternal ECG of Figure $8 \mathrm{~b}(\mathrm{i})$ is absent from Figure $8 \mathrm{~b}(\mathrm{ii})$. Furthermore, from a subjective perspective, the noise and FECG between the two figures have not been noticeably altered from the processing. The negligible change can be seen by visually comparing the FECG and surrounding noise of the encircled areas of 


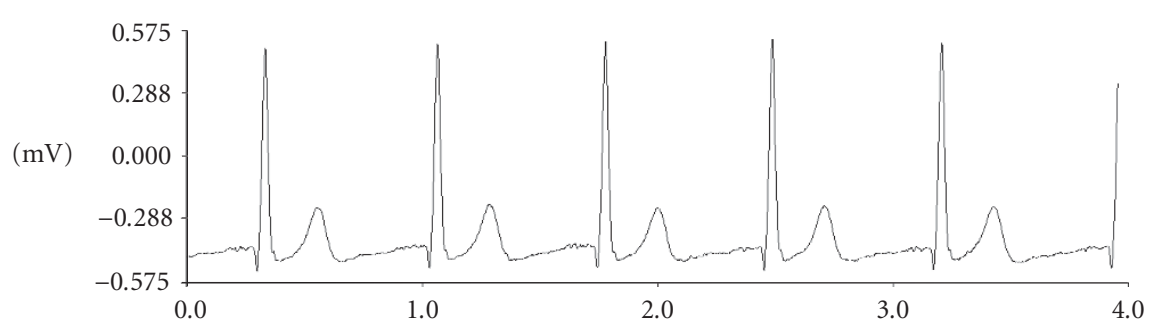

(a)

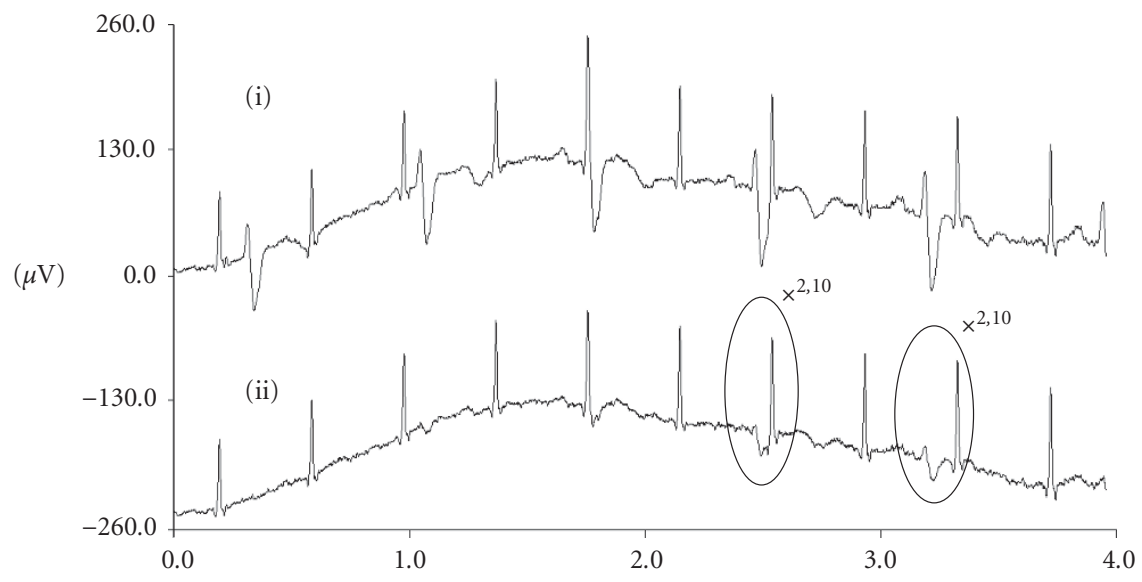

(b)

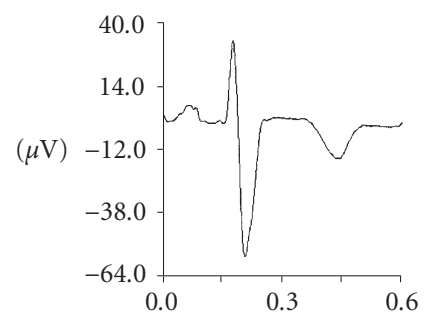

(c)

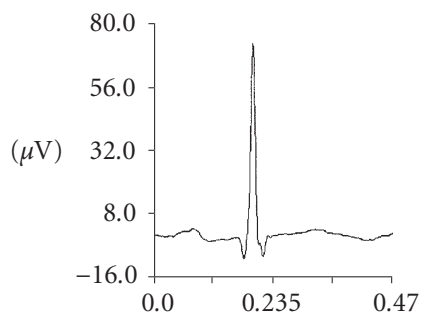

(d)

FIGURE 9: Intrauterine catheter synthesized data set 2 (all $x$-axes in seconds). (a) Thoracic ECG. (b)(i) Intrauterine catheter signal. (b)(ii) Intrauterine catheter signal with the maternal ECG canceled. (c) Maternal ECG average of the intrauterine catheter signal. (d) FECG average from the signal of (b)(ii) (55 complexes averaged).

Figures $8 \mathrm{~b}$ (i) and (ii) along with other portions of the two signals. Additional subjective analyses of Figures $8 \mathrm{~b}(\mathrm{i})$ and (ii) will be performed in Section 4.2.1.

Figure 8d is the pure FECG complex for comparison of the resulting FECG average in Figure 8e(i). The FECG average of Figure $8 \mathrm{e}(\mathrm{i})$ is from averaging 47 complexes. Figure $8 \mathrm{e}(\mathrm{ii})$ is the remaining noise still corrupting the FECG of Figure $8 \mathrm{e}(\mathrm{i})$. Figure $8 \mathrm{e}(\mathrm{ii})$ was obtained by subtracting the pure FECG complex of Figure 8d from the FECG average of Figure 8e(i). From a subjective point of view, the FECG average of Figure $8 \mathrm{e}(\mathrm{i})$ is similar to the pure FECG complex of Figure 8d, but with a small amount of noise that the processing introduced and did not remove. The processing introduced some noise to the final FECG average from small alignment errors during averaging [17]. The effects of alignment errors can be seen in Figure 8e(ii) as small spikes around the corresponding time location of the QRS complex of the signal of Figure $8 \mathrm{e}(\mathrm{i})$.

Figure $9^{1}$ demonstrates a condition where the proposed method breaks down. As with Figure 8 data, modifying IC clinical data was desired so that the resulting synthesized data would be as representative as possible of clinical data. Figure $9 a$ is the thoracic ECG. Figures $9 b$ (i) and $9 b$ (ii) are synthesized data via modulation of the clinical data of Figure $10 \mathrm{~b}(\mathrm{i})$ and resulting IC's ECG, respectively. The circles of Figure $9 \mathrm{~b}$ (ii) indicate the undesired maternal ECG

\footnotetext{
${ }^{1}$ The check marks $\times$ s and superscript numbers on this figure will be explained in Section 4.2.1.
} 


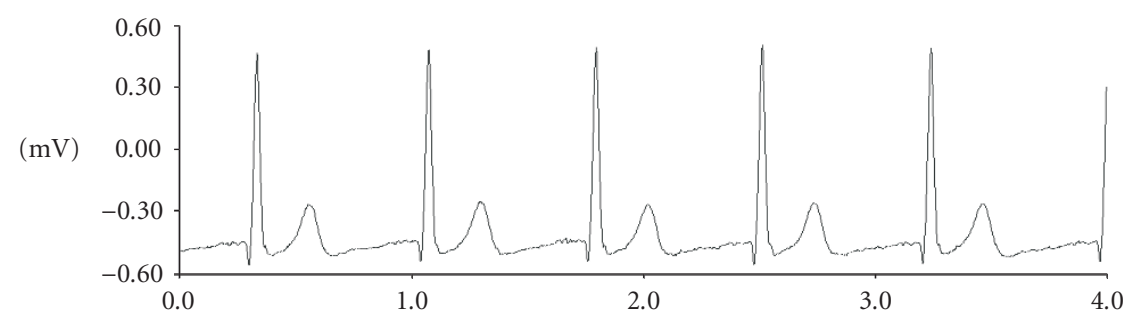

(a)

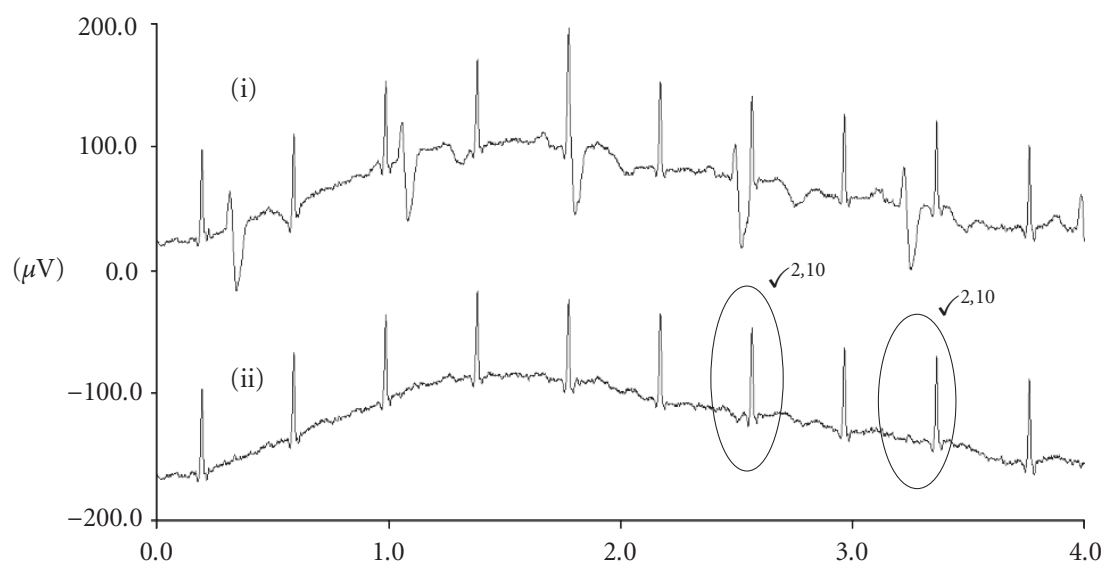

(b)

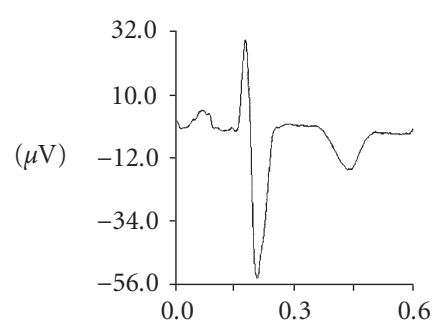

(c)

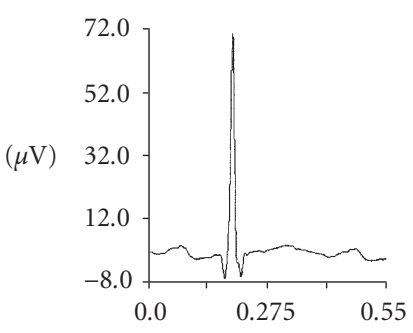

(d)

FIGURE 10: Intrauterine catheter clinical data from patient 1 (all $x$-axes in seconds). (a) Thoracic ECG. (b)(i) Intrauterine catheter signal. (b)(ii) Intrauterine catheter signal with the maternal ECG canceled. (c) Maternal ECG average of the intrauterine catheter signal. (d) FECG average from the signal of (b)(ii) (55 complexes averaged).

residuals. The ineffectiveness of the method arises from the modulated maternal ECG of the IC's ECG. Since the technique employs a maternal ECG average that has a fixed amplitude, significant variations in amplitude from modulation of the individual maternal ECG complexes of the IC's ECG can result in residuals during subtraction of the average.

\subsubsection{Objective analysis}

To qualify the subjective analysis or visual inspection, an objective analysis is necessary of the synthesized signals of Figure 8. A typical objective analysis of signal corruption will usually include signal-to-signal-plus-noise ratio, correlation coefficient, SNR, and other measures. The objective analysis of signal corruption for maternal ECG cancellation and FECG SNR enhancement includes signal-to-signal-plus- noise ratio, correlation coefficient, and SNR measures. The first corruption measure is defined by signal-to-signal-plusnoise ratio

$$
\mathrm{CM}_{1}=\frac{\sigma_{S_{\text {ideal }}(n)}^{2}}{\sigma_{S_{\text {ideal }}(n)}^{2}+\sigma_{N(n)}^{2}},
$$

where $\sigma_{S_{\text {ideal }}(n)}^{2}$ is the variance of $S_{\text {ideal }}(n)$ which is the desired untainted signal and $\sigma_{N(n)}^{2}$ is the variance of $N(n)$ which is the noise that contaminates $S_{\text {ideal }}(n)$. The second corruption measure is defined by correlation coefficient

$$
\mathrm{CM}_{2}=\frac{E\left[S_{\text {ideal }} R_{i}(n)\right]}{\sigma_{S_{\text {ideal }}(n)} \sigma_{R_{i}(n)}},
$$


where $R(n)$ is the resulting signal from the signal enhancement and $\sigma_{R(n)}^{2}$ is the variance of $R(n)$. The third corruption measure is defined by SNR

$$
\mathrm{CM}_{3}=\frac{\sigma_{S_{\text {ideal }}(n)}^{2}}{\sigma_{N(n)}^{2}} .
$$

A complete objective measure of IC maternal ECG cancellation cannot be performed because determining $S_{\text {ideal }}(n)$ is not possible from the fact that the pure maternal ECG of the IC's ECG of Figure 8b(i) cannot be determined. Artificial data could be used and this obstacle could be avoided, but the authenticity of the synthesized data would be greatly compromised, particularly, the relationship between the thoracic ECG and IC's ECG. A partial objective analysis of the signals of Figure $8 \mathrm{~b}$ is performed by analyzing the signal sections that do not have maternal ECG complexes that are marked with horizontal arrows. Since the proposed method for maternal ECG cancellation by definition does not process these parts of the IC's ECG, $\mathrm{CM}_{1}$ is $1.0, \mathrm{CM}_{2}$ is 1.0 , and $\mathrm{CM}_{3}$ is infinite. Corruption measures $\mathrm{CM}_{1}$ and $\mathrm{CM}_{3}$ indicate that the signal of these defined sections of the resulting IC's ECG have negligible signal corruption. Corruption measure $\mathrm{CM}_{2}$ denotes that the IC's ECG and resulting IC's ECG of these defined sections is completely correlated. A complete objective analysis of maternal ECG cancellation and FECG SNR enhancement is possible by using (6), (7), and (8) with $S_{\text {ideal }}(n)$ containing the pure FECG complexes of Figure $8 \mathrm{~d}, N(n)$ containing the signal of Figure $8 \mathrm{e}(\mathrm{ii})$, and $R(n)$ containing the signal of Figure $8 \mathrm{e}(\mathrm{i}) . \mathrm{CM}_{1}$ is $0.990, \mathrm{CM}_{2}$ is 0.995 , and $\mathrm{CM}_{3}$ is 109.0 or $1.0 \%$ of the signal energy of the FECG average of Figure 8e(i) is noise and the FECG average of Figure 8e(i) is 0.005 uncorrelated with the pure FECG complex of Figure $8 \mathrm{~d}$.

\subsection{Clinical data}

Clinical data was used to determine the effectiveness of the proposed algorithm. Several subjective visual inspections and one objective numerical measure that targets maternal ECG suppression were used to test the algorithm's effectiveness. The intent of the subjective analysis is to develop a rating system that will qualify the effectiveness of the method for maternal ECG cancellation. In addition, the purpose of the objective measure is to determine the average amount of maternal ECG suppression that can be expected from a variety of patients via the technique.

As previously discussed, clinical data was obtained from eight patients. Figures 10 through 17 present samples of this data. The subjective analysis was performed on the synthesized data of Figures 8 and 9 and the clinical data of Figures 10 through 17. Synthesized data was included with the clinical data study to reinforce the results from the clinical data and facilitate the explanation of the analyses. The objective analysis of maternal ECG cancellation was performed on the clinical data from the eight patients studied.

Since there are several figures referenced in this section, a template description of the figures is preferred to efficiently convey their layout format. Figures 10a through 17a present a thoracic ECG and Figures 10b(i) through 17b(i) present the IC's ECG. Figures $10 \mathrm{~b}$ (ii) through $17 \mathrm{~b}$ (ii) present the resulting IC's ECG with the maternal ECG canceled and Figures $10 \mathrm{c}$ through $17 \mathrm{c}$ present the maternal ECG average used to cancel the respective IC's maternal ECG of Figures $10 \mathrm{~b}(\mathrm{i})$ through $17 \mathrm{~b}(\mathrm{i})$. Some of the Figures 10 through 17 have a (d) graph. The (d) graph is the FECG average.

A discussion of each figure of data will not be performed, but a subjective analysis of the synthesized and clinical data of this section will be presented in a tabular format. The tabular format was used to efficiently illustrate the subjective results of the method for maternal ECG cancellation. Finally, the objective measure of the average amount of maternal ECG suppression that can be expected with the proposed technique will be discussed.

\subsubsection{Subjective analysis}

The subjective analysis examines synthesized and clinical data from eight patients. The subjective analysis can be divided into the engineering aspects and clinical applicability analyses. The Engineering aspect analysis examines the original and resulting IC's ECGs, by visual inspection, to determine whether the proposed method for maternal ECG cancellation passes or fails 10 test conditions that are based on signal characteristics. The clinical applicability analysis examines the resulting IC's ECG, also by visual inspection, to determine whether the method for maternal ECG cancellation produced a potentially or not potentially clinically useful FECG for three different clinical applications. For the engineering aspect analysis, a ratio of success rating from the grading of each of the 10 test conditions is determined for the clinical data. For the clinical applicability analysis, a clinical potential rating from the grading of each of the three clinical applications is determined for the clinical data. Tables 2 and 3 present the grading of maternal ECG attenuation for the engineering aspect and clinical applicability analyses for the synthesized data and clinical data of Figures 8 through 17. Table 3 also presents the ratio of success and clinical potential ratings for the clinical data.

Example explanations will be presented with the synthesized and clinical data of Figures 9 and 10 to further clarify the grading of the test conditions and clinical applications. Furthermore, synthesized data in comparison with the clinical data demonstrates more conclusively the grading of some of the test conditions. Figures 9 and 10 are identical, but the IC's ECG of Figure $9 \mathrm{~b}$ (i) has been synthesized via modulating the maternal ECG of the clinical data of Figure 10b(i). Since the applicable test conditions of Figure 10 data were all reported pass as indicated in Table 2 , any observed failures in Figure 9 can be attributed to the modulated maternal ECG.

\section{Engineering aspects}

For Figures 9 and 10, test condition 1 was graded a pass because the resulting IC's ECGs from maternal ECG cancellation by definition are 0.05 to $100.0 \mathrm{~Hz}$. See Section 3 for method description. 
TABLE 2: Inspection results for maternal ECG cancellation effectiveness of Figures 8 to 12.

\begin{tabular}{|c|c|c|c|c|c|}
\hline \multicolumn{6}{|c|}{ Engineering aspects } \\
\hline Test conditions & & Data des & and gradin & ation & \\
\hline Possible outcomes & Figure 8 & Figure 9 & Figure 10 & Figure 11 & Figure 12 \\
\hline$\checkmark$ Passed $\times$ Failed & Synthesized & Synthesized & Patient 1 & Patient 2 & Patient 3 \\
\hline — Did not occur & FECG & FECG & FECG & FECG & FECG \\
\hline $\begin{array}{l}1 \text { Bandwidth of resulting IC's } \\
\text { ECG is } 0.05 \text { to } 100.00 \mathrm{~Hz}\end{array}$ & $\checkmark$ & $\checkmark$ & $\checkmark$ & $\checkmark$ & $\checkmark$ \\
\hline $\begin{array}{l}\text { Signal corruption to FECG } \\
\text { \& EMG }\end{array}$ & $\checkmark$ & $\times^{10^{*}}$ & $\checkmark$ & $\checkmark$ & $\checkmark$ \\
\hline $\begin{array}{l}\text { Overall maternal ECG } \\
\text { cancellation }\end{array}$ & $\checkmark$ & $\checkmark$ & $\checkmark$ & $\checkmark$ & $\checkmark$ \\
\hline $\begin{array}{l}4 \text { FECG \& maternal ECG } \\
\text { overlap }\end{array}$ & $\checkmark$ & $\checkmark$ & $\checkmark$ & $\checkmark$ & $\checkmark$ \\
\hline $\begin{array}{ll}5 & \text { Atypically shaped maternal } \\
& \text { ECG of the IC } \\
\end{array}$ & - & - & - & $\checkmark$ & - \\
\hline $\begin{array}{l}6 \text { Significant IC low-frequency } \\
\text { noise }\end{array}$ & $\checkmark$ & $\checkmark$ & $\checkmark$ & $\checkmark$ & - \\
\hline $7 \quad$ Significant IC noise spike & $\checkmark$ & - & - & - & - \\
\hline $\begin{array}{ll}8 & \text { Thoracic ECG random } \\
\text { and/or } 60 \mathrm{~Hz} \text { noise }\end{array}$ & - & - & - & $\checkmark$ & $\checkmark$ \\
\hline $\begin{array}{ll}9 & \text { IC random and/or } 60 \mathrm{~Hz} \\
& \text { noise } \\
\end{array}$ & $\checkmark$ & $\checkmark$ & $\checkmark$ & $\checkmark$ & $\checkmark$ \\
\hline $\begin{array}{l}10 \text { Modulated maternal ECG of } \\
\text { the IC }\end{array}$ & - & $\times$ & - & - & - \\
\hline
\end{tabular}

\begin{tabular}{|c|c|c|c|c|c|}
\hline \multicolumn{6}{|c|}{ Clinical applicability } \\
\hline Clinical applications & & Data d & and gradin & tion & \\
\hline P Potential NP No potential & Figure 8 & Figure 9 & Figure 10 & Figure 11 & Figure 12 \\
\hline $\begin{array}{l}1 \text { Fetal HR information } \\
\text { via HR determination } \\
\text { algorithm }\end{array}$ & $\mathrm{P}$ & $\mathrm{P}$ & $\mathrm{P}$ & $\mathrm{P}$ & $\mathrm{P}$ \\
\hline $\begin{array}{l}2 \text { FECG electrophysiological } \\
\text { information via FECG } \\
\text { averaging }\end{array}$ & $\mathrm{P}$ & $\mathrm{P}$ & $\mathrm{P}$ & $\mathrm{P}$ & $\mathrm{P}$ \\
\hline $\begin{array}{l}3 \text { Uterine contraction } \\
\text { information via } \\
\text { EMG }\end{array}$ & $\mathrm{P}$ & NP & $\mathrm{P}$ & $\mathrm{P}$ & $\mathrm{P}$ \\
\hline
\end{tabular}

*The failure of test condition 10 caused test conditions 2 to fail.

Test condition 2 was graded fail and pass for Figures 9 and 10, respectively, which was determined from a visual inspection of the two resulting IC's ECGs. The resulting IC's ECGs compared to the FECG or EMG of the IC's ECGs had noticeable and negligible maternal ECG residuals for Figures 9 and 10, respectively. The $\times$ with superscript ten of Table 2, test condition 2 row and Figure 9 column, indicates that test condition 2 was reported a failure because test condition 10 failed. Furthermore, the $\times$ and $\checkmark$ with superscripts two and ten indicate the locations of noticeable and negligible maternal ECG residuals that caused test condition 2 to be reported fail and pass of Figures $9 \mathrm{~b}$ (ii) and $10 \mathrm{~b}$ (ii), respectively. Test condition 2 will be reported a failure when any of the test conditions 5 through 10 fail.

Figures 9 and 10 had two and zero, respectively, out of five maternal ECG complexes resulting in noticeable maternal ECG residuals compared to the FECG and EMG of the IC's ECG. Since both figures had noticeable residuals less than the majority of maternal ECG complexes, test condition 3 was passed for Figures 9 and 10. 
TABLE 3: Inspection results for maternal ECG cancellation effectiveness of Figures 13 to 17.

\begin{tabular}{|c|c|c|c|c|c|c|}
\hline \multicolumn{7}{|c|}{ Engineering aspects } \\
\hline Test conditions & \multicolumn{6}{|c|}{ Data designation and grading information } \\
\hline Possible outcomes & Figure 13 & Figure 14 & Figure 15 & Figure 16 & Figure 17 & Ratio \\
\hline$\checkmark$ Passed $\times$ Failed & Patient 4 & Patient 5 & Patient 6 & Patient 7 & Patient 8 & of \\
\hline — Did not occur & Weak FECG & No FECG & FECG & FECG & FECG & Success \\
\hline $\begin{array}{l}1 \text { Bandwidth of resulting IC's } \\
\text { ECG is } 0.05 \text { to } 100.00 \mathrm{~Hz}\end{array}$ & $\checkmark^{*}$ & $\checkmark$ & $\checkmark$ & $\checkmark$ & $\checkmark$ & $8 / 8$ \\
\hline $\begin{array}{l}2 \text { Signal corruption to FECG } \\
\text { \& EMG }\end{array}$ & $\checkmark$ & $\checkmark$ & $\times^{10^{* *}}$ & $\checkmark$ & $\checkmark$ & $6 / 8$ \\
\hline $\begin{array}{l}\text { Overall maternal ECG } \\
\text { cancellation }\end{array}$ & $\checkmark$ & $\checkmark$ & $\times^{10^{* *}}$ & $\checkmark$ & $\checkmark$ & $7 / 8$ \\
\hline $\begin{array}{l}4 \text { FECG \& maternal ECG } \\
\text { overlap }\end{array}$ & $\checkmark$ & - & - & $\checkmark$ & $\checkmark$ & $6 / 6$ \\
\hline $\begin{array}{l}5 \text { Atypically shaped maternal } \\
\text { ECG of the IC }\end{array}$ & $\checkmark$ & - & - & $\checkmark$ & $\checkmark$ & $3 / 3$ \\
\hline $\begin{array}{l}\text { Significant IC low-frequency } \\
\text { noise }\end{array}$ & $\checkmark$ & $\checkmark$ & - & $\checkmark$ & - & $5 / 5$ \\
\hline $7 \quad$ Significant IC noise spike & - & $\checkmark$ & - & - & - & $1 / 1$ \\
\hline $\begin{array}{ll}8 & \text { Thoracic ECG random } \\
\text { and/or } 60 \mathrm{~Hz} \text { noise }\end{array}$ & - & - & $\checkmark$ & $\checkmark$ & $\checkmark$ & $5 / 5$ \\
\hline $\begin{array}{ll}9 & \text { IC random and/or } 60 \mathrm{~Hz} \\
\text { noise }\end{array}$ & $\checkmark$ & $\checkmark$ & $\checkmark$ & $\checkmark$ & $\checkmark$ & $8 / 8$ \\
\hline $\begin{array}{l}10 \text { Modulated maternal ECG of } \\
\text { the IC }\end{array}$ & - & - & $\times$ & - & - & $0 / 1$ \\
\hline \multicolumn{7}{|c|}{ Clinical applicability } \\
\hline Clinical applications & \multicolumn{6}{|c|}{ Data designation and grading information } \\
\hline P Potential NP No potential & Figure 13 & Figure 14 & Figure 15 & Figure 16 & Figure 17 & Potentials \\
\hline $\begin{array}{l}1 \text { Fetal HR information via } \\
\text { HR determination } \\
\text { algorithm }\end{array}$ & $\mathrm{P}$ & NP & $\mathrm{P}$ & $\mathrm{P}$ & $\mathrm{P}$ & $7 / 8$ \\
\hline $\begin{array}{l}2 \text { FECG electrophysiological } \\
\text { information via FECG } \\
\text { averaging }\end{array}$ & $\mathrm{NP}$ & $\mathrm{NP}$ & $\mathrm{P}$ & $\mathrm{P}$ & $\mathrm{P}$ & $6 / 8$ \\
\hline $\begin{array}{l}3 \text { Uterine contraction } \\
\text { information via } \\
\text { EMG }\end{array}$ & $\mathrm{P}$ & $\mathrm{P}$ & $\mathrm{NP}$ & $\mathrm{P}$ & $\mathrm{P}$ & $7 / 8$ \\
\hline $\begin{array}{l}\text { *The IC's ECG bandwidth was set } \\
\text { instrumentation even at low-gain } \\
\text { test condition } 1 \text { was passed. }\end{array}$ & $\begin{array}{l}\text { to } 100.00 \mathrm{~Hz} \\
\text { gs. The } 0.5 \text { to }\end{array}$ & $\begin{array}{l}\text { excessive } 1 \\
\text { Iz bandwidt }\end{array}$ & $\begin{array}{l}\text { quency noi } \\
\text { not a requi }\end{array}$ & $\begin{array}{l}\text { at would sat } \\
\text { nt of mater }\end{array}$ & $\begin{array}{l}\text { the output } \\
\text { CG cancella }\end{array}$ & $\begin{array}{l}\text { front-end } \\
\text { Therefore, }\end{array}$ \\
\hline
\end{tabular}

Test conditions 4 was awarded a pass grade for Figures 9 and 10 since the FECG's QRS complex that overlapped the maternal ECG's QRS complex of the IC's ECG is present and similar in shape to the surrounding nonoverlapping FECG's QRS complexes in the resulting IC's ECG.

An atypically shaped maternal ECG of the IC's ECG did not occur in Figures 9 and 10. An atypically shaped maternal
ECG of the IC's ECG would be an ECG signal that does not have the shape of an adult ECG obtained from a lead I, II, or III position, which represent standardized ECG electrode positions. In addition, the IC's ECGs of Figures 9 and 10 did not have a significant noise spike or thoracic ECG random and/or $60 \mathrm{~Hz}$ noise. Therefore, test conditions 5, 7, and 8 that were graded did not occur. Figure 11 contains an atypically 


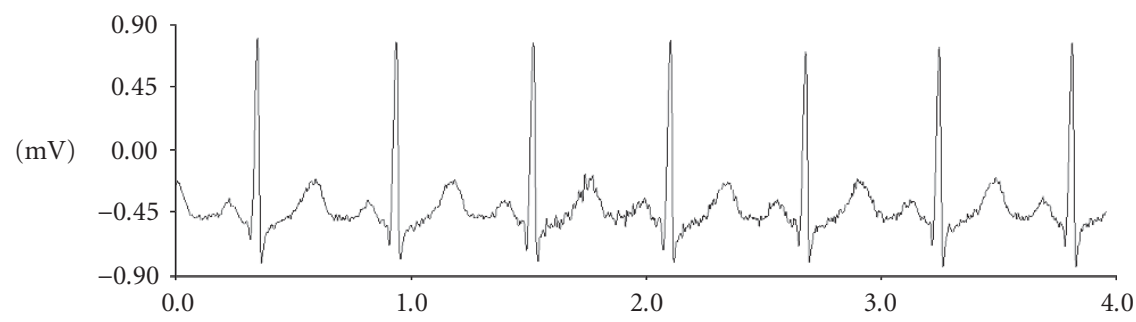

(a)

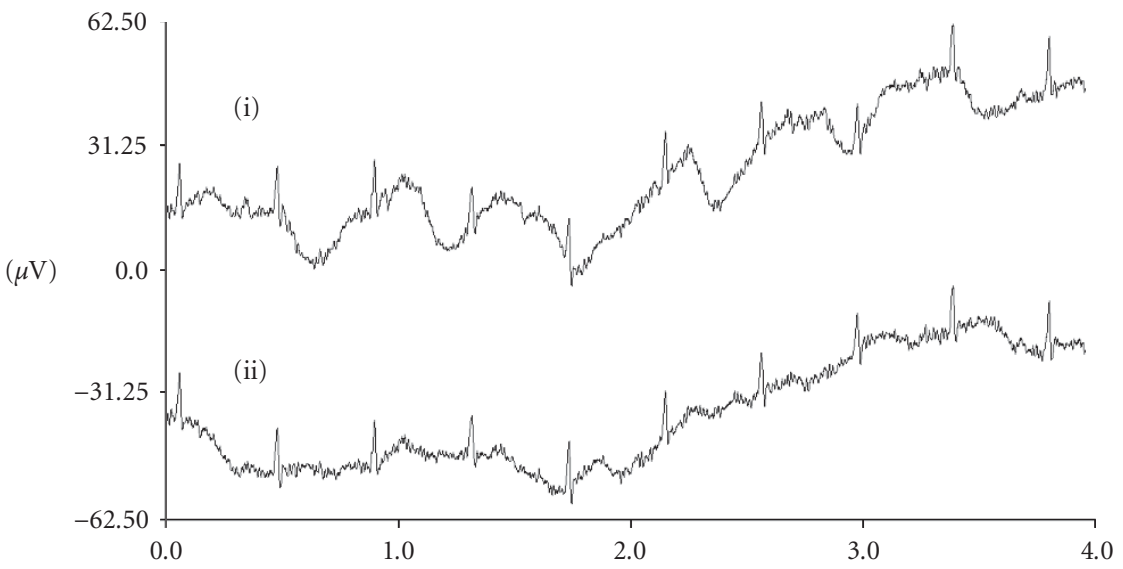

(b)

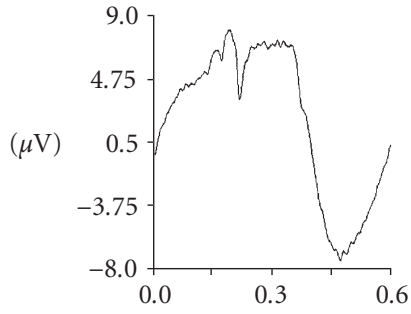

(c)

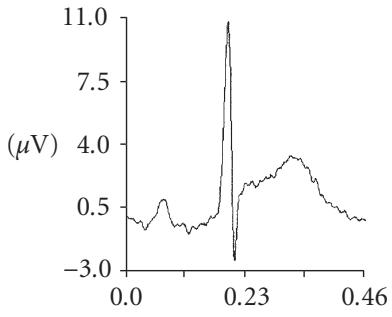

(d)

FIGURE 11: Intrauterine catheter clinical data from patient 2 (all $x$-axes in seconds). (a) Thoracic ECG. (b)(i) Intrauterine catheter signal. (b)(ii) Intrauterine catheter signal with the maternal ECG canceled. (c) Maternal ECG average of the intrauterine catheter signal. (d) FECG average from the signal of (b)(ii) (50 complexes averaged).

shaped maternal ECG of the IC's ECG and thoracic ECG random noise. Figure 8 is an example of a noise spike in the IC's ECG.

Figures 9 and 10 contain significant IC's ECG lowfrequency noise and random IC's ECG noise. Figure 9 does contain a significant maternal ECG residual which has already been determined to be from the modulated maternal ECG of the IC's ECG. Therefore, test conditions 6 and 9 were awarded pass grades for Figures 9 and 10.

Figure 9 has a modulated maternal ECG of the IC's ECG that has resulted in the IC's ECG compared to the FECG or EMG of the IC's ECG to have a noticeable maternal ECG residual. Therefore, test condition 10 was awarded a fail grade. Since Figure 10 did not have a modulated maternal ECG of the IC's ECG, test condition 10 was awarded a did not occur grade.

\section{Engineering aspects ratio of success}

The ratio of success is a measure of the success of the 10 test conditions for the clinical data. The ratios are determined by computing the number of passes for each test condition and dividing it by the number of occurrences of each test condition. The ratios at the top of the last column of Table 3 are the ratios of success for the test conditions for the eight patients studied.

\section{Clinical applicability}

The first clinical application pertains to fetal HR information via a HR determination algorithm. Potential grades were awarded to Figures 9 and 10. A potential grade was awarded for Figures 9 and 10 since the resulting IC's ECG had an observable FECG as well as test condition 3 was graded pass. 


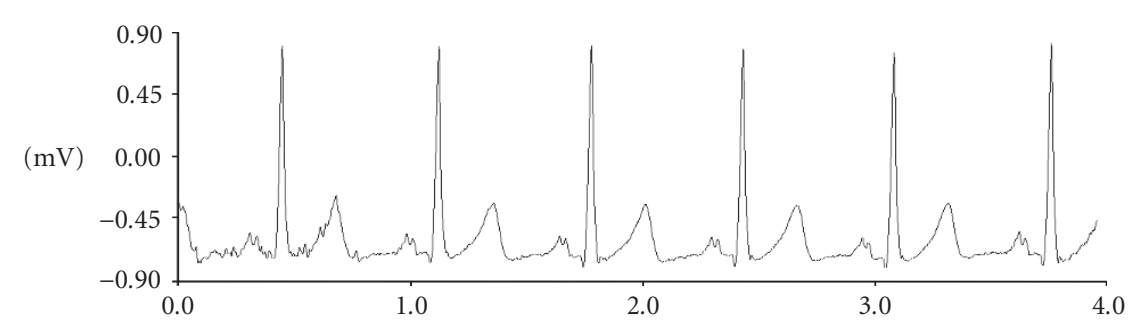

(a)

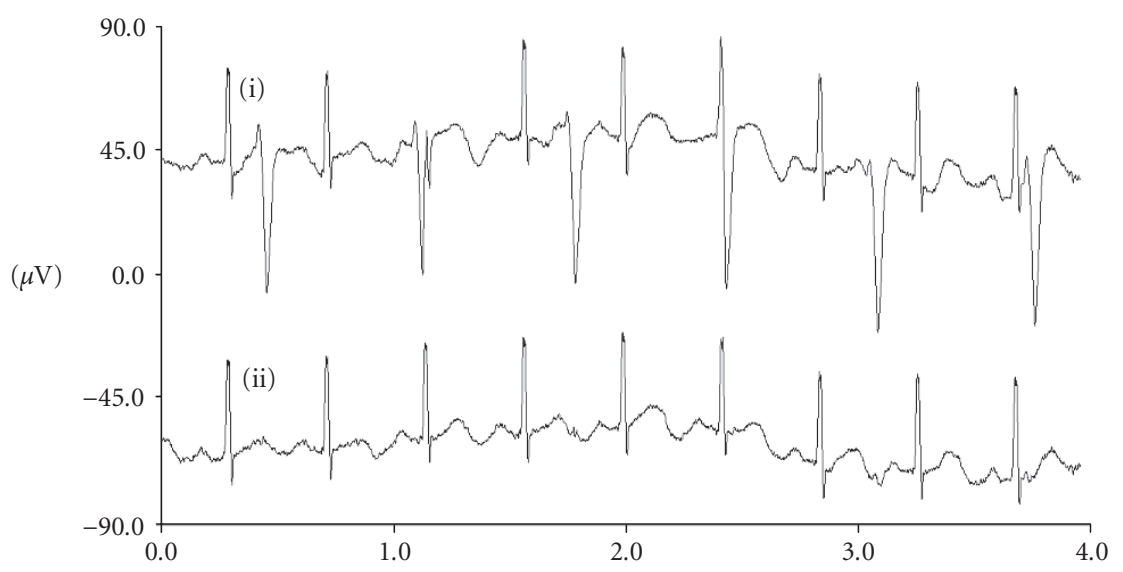

(b)

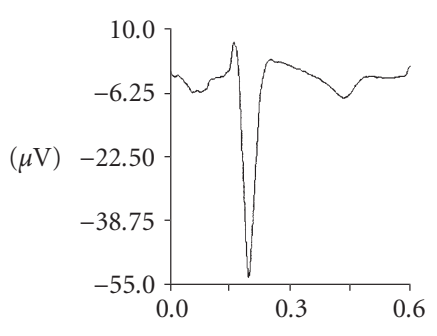

(c)

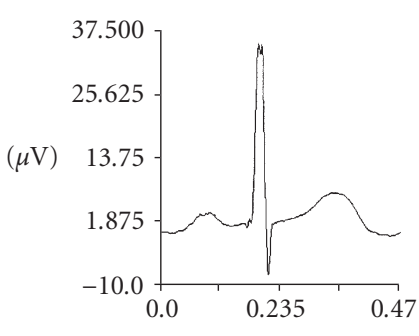

(d)

FIGURE 12: Intrauterine catheter clinical data from patient 3 (all $x$-axes in seconds). (a) Thoracic ECG. (b)(i) Intrauterine catheter signal. (b)(ii) Intrauterine catheter signal with the maternal ECG canceled. (c) Maternal ECG average of the intrauterine catheter signal. (d) FECG average from the signal of (b)(ii) (49 complexes averaged).

The second clinical application pertains to the determination of the FECG electrophysiological information via FECG averaging. Potential grades were awarded to Figures 9 and 10. A potential grade was awarded for Figures 9 and 10 since test conditions 1 and 3 passed. Furthermore, FECG averaging has potential to produce a FECG average with a favorable SNR since a visual inspection of the resulting IC's ECG of Figures 9b(ii) and 10b(ii) can discern all FECG's R waves to be averaged.

The final clinical application is uterine contraction information via EMG. This test determines if the EMG signal of the resulting IC's ECG has been corrupted by maternal ECG cancellation. If the EMG is not tainted, then uterine contraction information can be accurately derived from the EMG and a potential status is awarded. If the EMG is tainted, then uterine contraction information cannot be accurately derived from the EMG and a no potential grade is awarded. The grading of potential and no potential is an indicator that the EMG is undistorted or distorted, respectively. In addition, the grading of potential and no potential should not be interpreted as indicators for uterine contraction information being present or not present, respectively. The data of Figure 9 has no potential to determine uterine contraction information since test condition 2 failed. The data of Figure 10 has potential to determine uterine contraction information since test conditions 1 and 2 passed.

\section{Clinical applicability potentials}

The ratio of potentials is a measure of the clinical usefulness of the resulting IC's ECG for three clinical applications for 


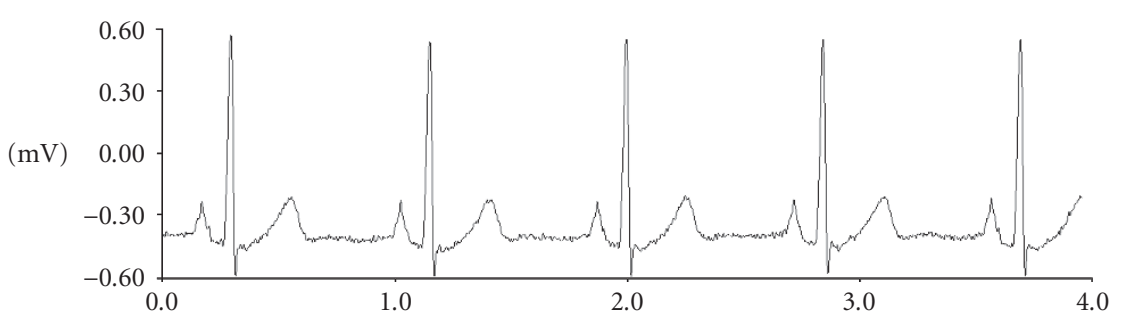

(a)

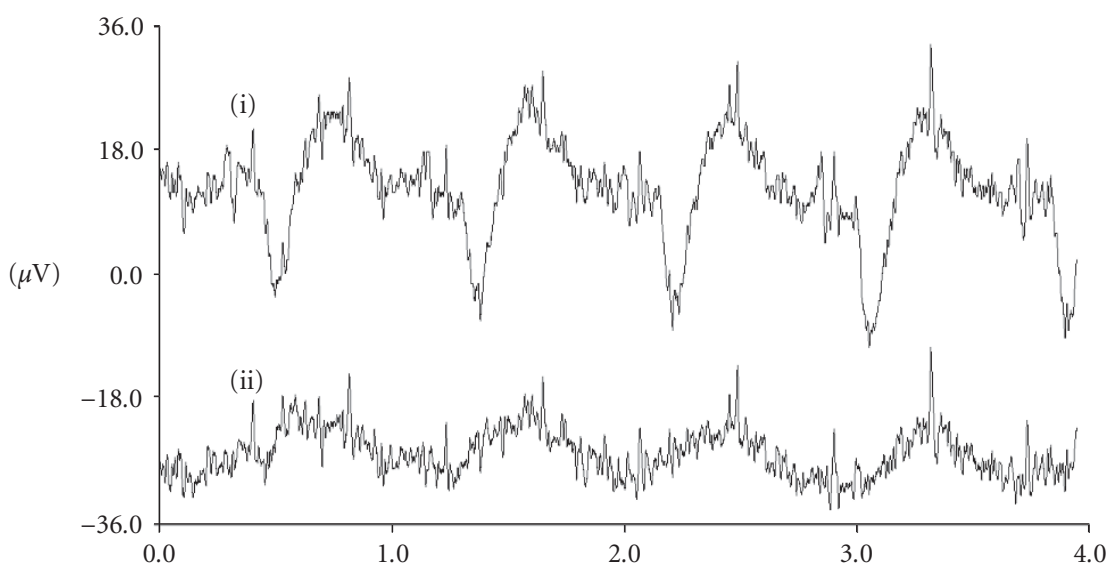

(b)

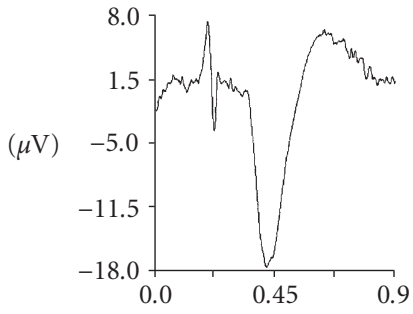

(c)

FIGURE 13: Intrauterine catheter clinical data from patient 4 (all $x$-axes in seconds). (a) Thoracic ECG. (b)(i) Intrauterine catheter signal. (b)(ii) Intrauterine catheter signal with the maternal ECG canceled. (c) Maternal ECG average of the intrauterine catheter signal.

the clinical data. The ratios are determined by computing the number of potentials for each clinical application and dividing it by the total number of clinical data sets studied. The ratios at the bottom of the last column of Table 3 are the ratios of potential for each clinical application for the eight patients studied.

\subsubsection{Objective analysis}

The objective analysis of the clinical data consists of a statistical computation that determines the expected (mean) value of maternal ECG suppression with the proposed method if the reader were to implement the cancellation algorithm. An analysis of eight patients with a variety of signal characteristics was performed to obtain a representative expected value.
The analysis measures the maternal ECG suppression of the IC's ECG for 24 continuous maternal ECG complexes for each of the eight patients. The maternal ECG suppression was determined by measuring the peak-to-peak values of each maternal ECG complex of the IC's ECG and resulting IC's ECG at the time location corresponding to the maternal ECG complex of the IC's ECG. Then the peak-to-peak value of the maternal ECG complex of the IC's ECG is divided by the peak-to-peak value of the corresponding resulting IC's ECG residual to obtain the maternal ECG suppression ratio for that particular maternal ECG complex. The expected or mean value of maternal ECG suppression was then found by summing all the suppression ratios and dividing by 192 . The expected value was calculated to be $8.8 \mathrm{~V} / \mathrm{V}$. The standard deviation of the suppression ratios was found to be $2.4 \mathrm{~V} / \mathrm{V}$. 


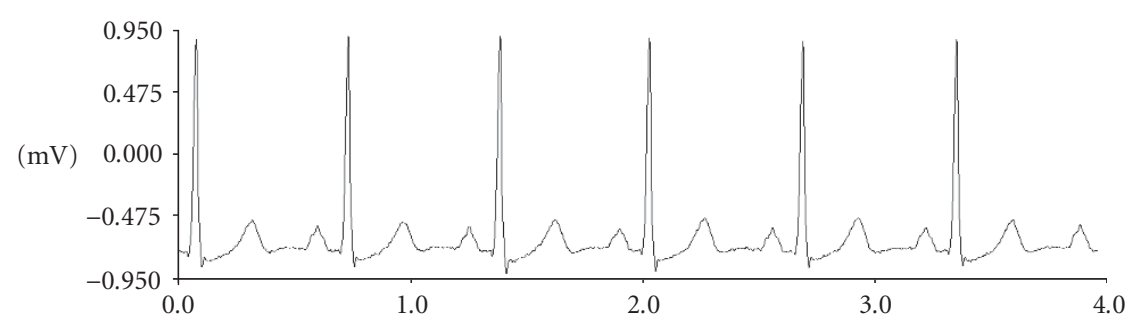

(a)

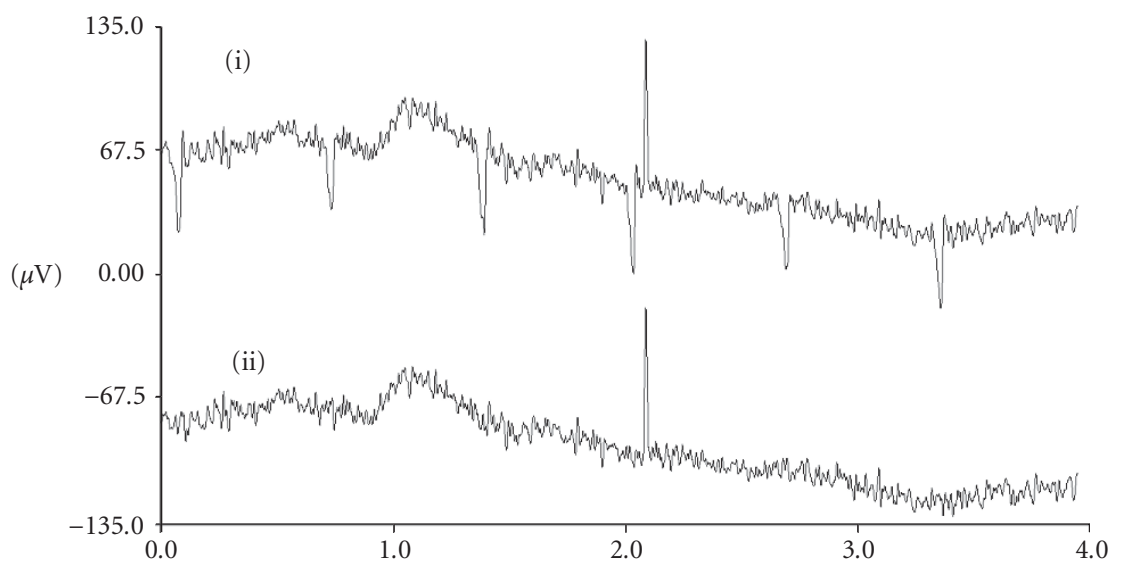

(b)

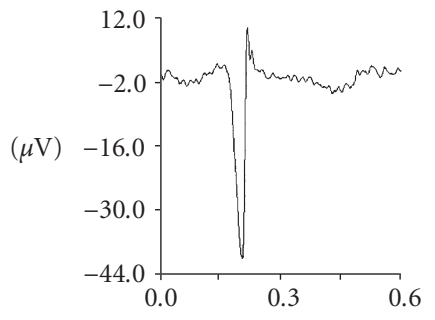

(c)

FIGURE 14: Intrauterine catheter clinical data from patient 5 (all $x$-axes in seconds). (a) Thoracic ECG. (b)(i) Intrauterine catheter signal. (b)(ii) Intrauterine catheter signal with the maternal ECG canceled. (c) Maternal ECG average of the intrauterine catheter signal.

\subsection{Discussion}

From the subjective visual inspection of synthesized data of Figure 8, the resulting IC's ECG had no noticeable alterations compared to the IC's ECG from maternal ECG cancellation. In addition, the FECG average had discernible P, QRS, and $\mathrm{T}$ waves and negligible differences when compared to the pure FECG complex. The objective corruption measures qualify, with numerical values, the subjective visual inspection. Since resulting values for $\mathrm{CM}_{1}, \mathrm{CM}_{2}$, and $\mathrm{CM}_{3}$ for maternal ECG cancellation indicated no change to the resulting IC's compared to the IC's ECG, these three corruption measures are considered favorable. Furthermore, since the FECG average contained less than $1 \%$ of noise and had fully discernible $\mathrm{P}, \mathrm{QRS}$, and $\mathrm{T}$ waves, these results indicate that the three corruption measures for maternal ECG cancellation and FECG SNR enhancement are also favorable. Therefore, the analysis verifies that the method has performed effectively with synthesized data and has significant potential to enhance clinical data accurately.

Since an objective analysis similar to the one performed with synthesized data cannot be achieved with clinical data, a subjective analysis was performed. This analysis qualifies the effectiveness of the proposed maternal ECG cancellation algorithm with clinical data via summarizing the results of the ten test conditions in a percentage success specification. Table 4 contains the percentage successes.

Eight of the ten test conditions scored greater than $87.5 \%$ and seven of the ten scored $100 \%$. Considering that the data sets contain ideal, typical, and nonideal data, the scores of 


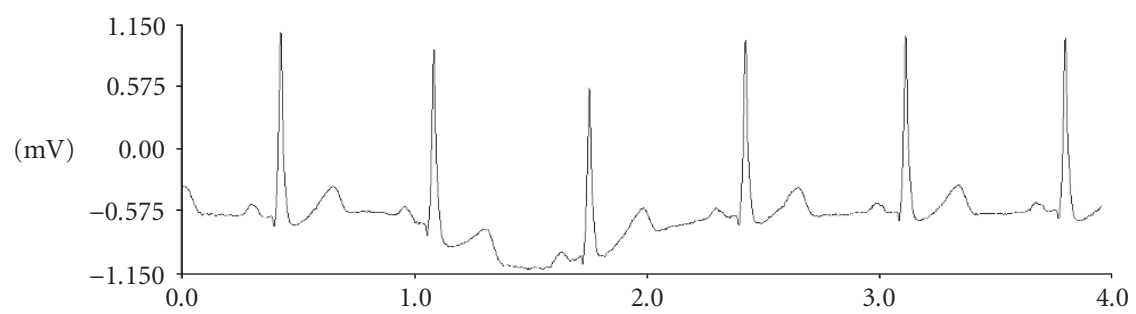

(a)

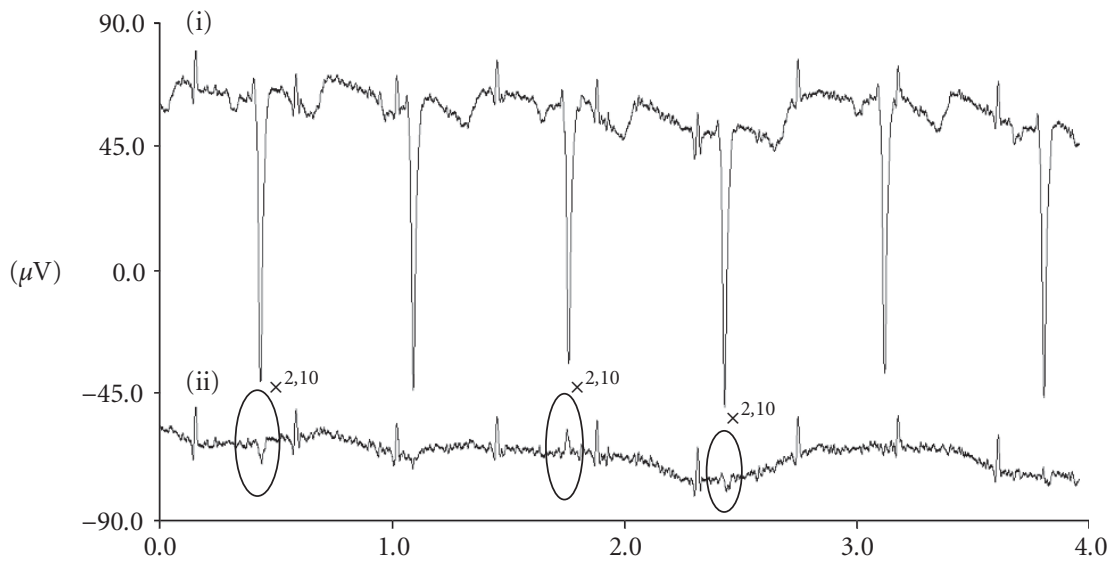

(b)

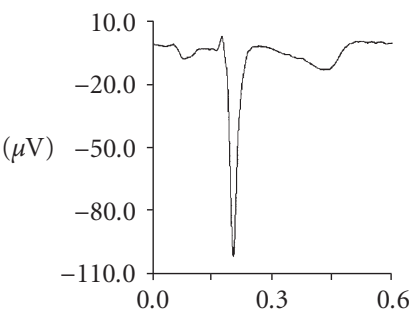

(c)

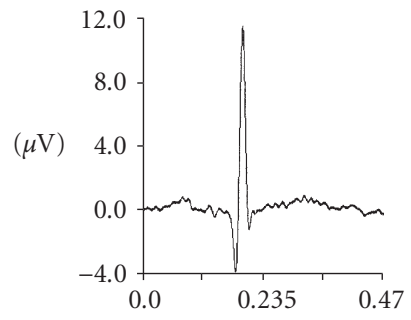

(d)

FIGURE 15: Intrauterine catheter clinical data from patient 6 (all $x$-axes in seconds). (a) Thoracic ECG. (b)(i) Intrauterine catheter signal. (b)(ii) Intrauterine catheter signal with the maternal ECG canceled. (c) Maternal ECG average of the intrauterine catheter signal. (d) FECG average from the signal of (b)(ii) (69 complexes averaged).

greater than $87.5 \%$ for eight of the ten tests suggest that the maternal ECG cancellation algorithm is performing effectively for these test conditions. Furthermore, these eight test conditions also indicate the strengths of the method. The scores of $75 \%$ and $0 \%$ of test conditions 2 and 10 , respectively, suggest that improvements can still be made to the maternal ECG cancellation algorithm. Test condition 2 is the most difficult test condition of the ten since it is dependent on a passing grade from test conditions 5 through 10. The one failure of test condition 2 was directly related to test condition 10 , which indicates a weakness in the maternal ECG cancellation algorithm during maternal ECG modulation.

Two different approaches were studied to reduce the ma- ternal ECG modulation residual. The first approach determined a gain factor for each maternal ECG average for subtraction. The gain factor would allow the amplitude of the maternal ECG average to be closely matched to each maternal ECG complex for subtraction. However, since the maternal ECG complex and FECG complex can be significantly correlated in certain cases of overlap, the gain factor of the maternal ECG average can be influenced by the correlation between the two complexes. The third maternal ECG complex of Figure 10b(i) demonstrates overlap where the two complexes are significantly correlated. The correlation influences the gain factor such that some of the FECG complex is canceled during subtraction of the average, which causes test condition 4 to fail. 


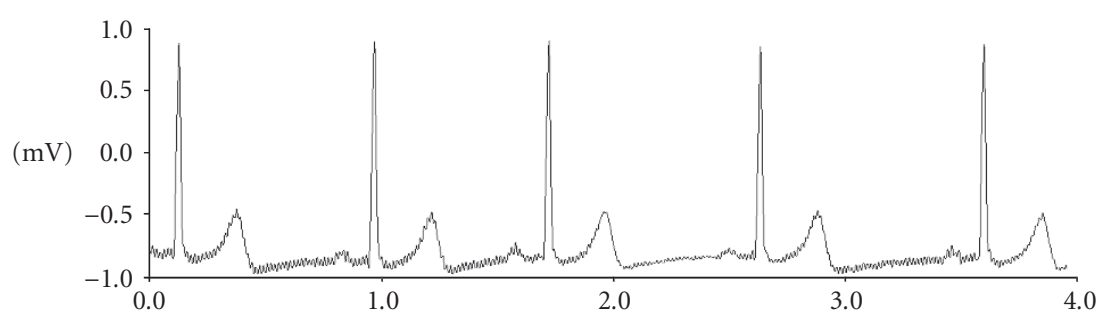

(a)

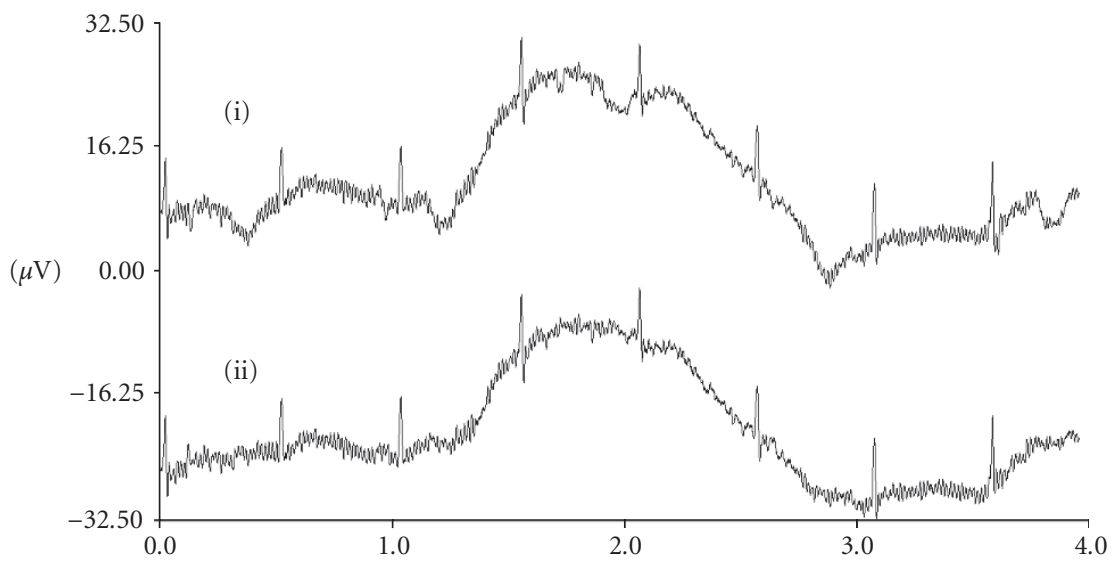

(b)

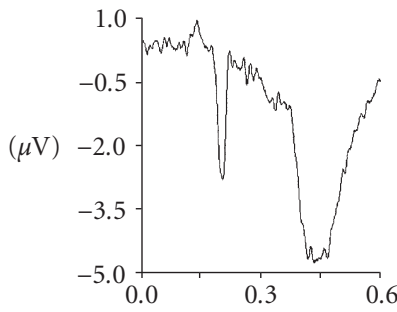

(c)

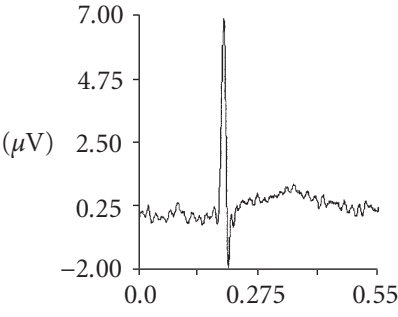

(d)

FIGURE 16: Intrauterine catheter clinical data from patient 7 (all $x$-axes in seconds). (a) Thoracic ECG. (b)(i) Intrauterine catheter signal. (b)(ii) Intrauterine catheter signal with the maternal ECG canceled. (c) Maternal ECG average of the intrauterine catheter signal. (d) FECG average from the signal of (b)(ii) (42 complexes averaged).

Since the FECG is the desired, another approach is preferred. The second approach would be to reduce the residual from modulation during FECG SNR enhancement by FECG averaging, adaptive filtering, or other FECG SNR enhancement techniques $[5,10,11]$. Finally, a good future research topic would be to develop an intelligent algorithm that accurately detects when a FECG complex overlaps with the maternal ECG complexes and apply an adaptive gain control algorithm to reduce the residual from a modulated maternal ECG when the maternal and FECG complexes do not overlap. To avoid any FECG cancellation from correlation between the maternal ECG and FECG complexes during overlap, the adaptive gain control algorithm would not be used for complexes that overlap.
In addition to the modulated maternal ECG, maternal ECG complexes that have abnormal shapes can also result in a residual. A preventicle contraction will produce an abnormal complex which consists of an unexpected QRS wave that does not include a $\mathrm{P}$ or $\mathrm{T}$ wave. The extra QRS wave will occur in both the thoracic and IC's ECGs and will be averaged out with the other detected maternal ECG complexes. Since the maternal ECG complex from the preventicle contraction does not include $\mathrm{P}$ and $\mathrm{T}$ waves, a residual results when the maternal ECG average that contains the P, QRS, and T waves is subtracted from the IC's ECG. Preventicle contractions or other abnormal maternal ECG complex did not occur in the eight patient studied. However, a few preventicle contractions did occur for one of the 17 patients we studied from 


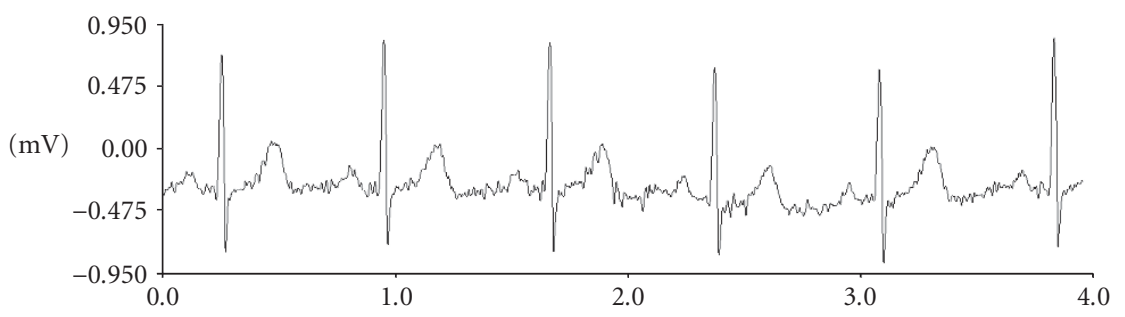

(a)

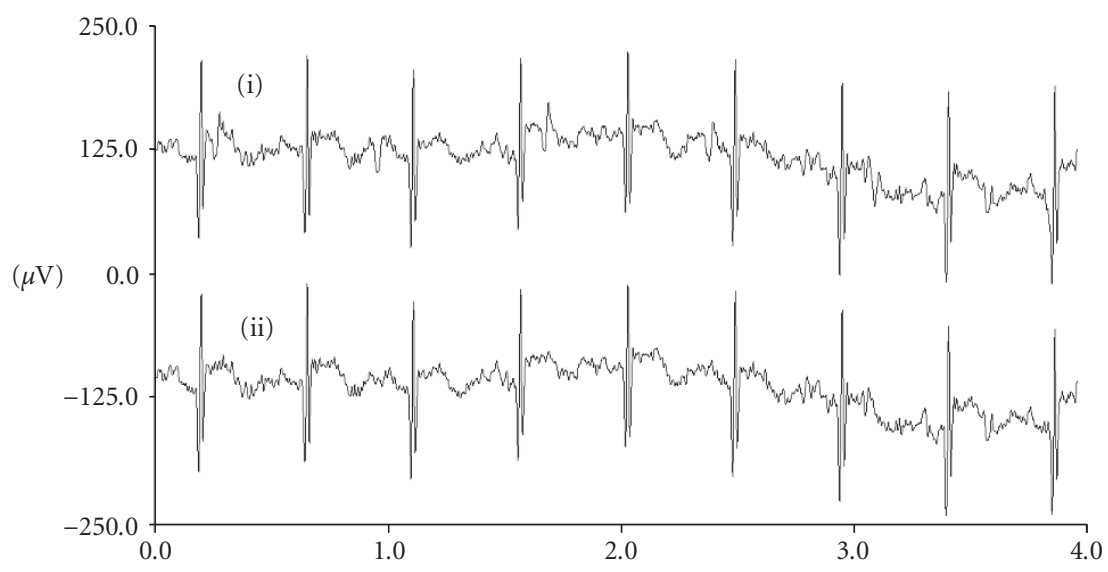

(b)

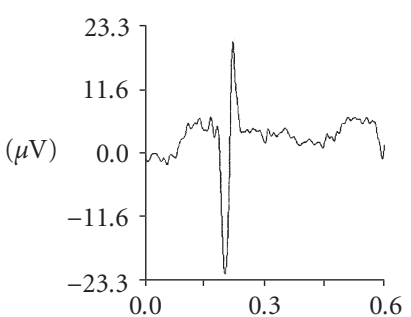

(c)

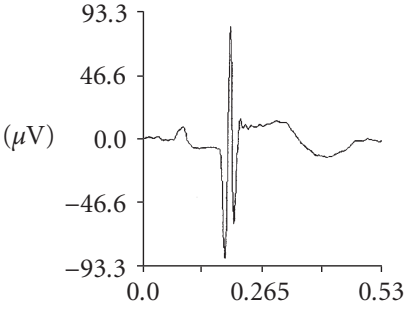

(d)

FIGURE 17: Intrauterine catheter clinical data from patient 8 (all $x$-axes in seconds). (a) Thoracic ECG. (b)(i) Intrauterine catheter signal. (b)(ii) Intrauterine catheter signal with the maternal ECG canceled. (c) Maternal ECG average of the intrauterine catheter signal. (d) FECG average from the signal of (b)(ii) (45 complexes averaged).

a previous research project for noninvasive abdominal wall FECG enhancement. Future research is necessary to address the occurrence of abnormal maternal ECG complexes.

Table 5 contains the percentage potentials of the three clinical applications. Percentage potentials of $87.5 \%$ and $75 \%$ for fetal HR and FECG electrophysiological information, respectively, indicate some limitation of the IC approach. This restriction is directly related to the FECG's signal strength because the potential grading is highly dependent on a positive visual identification of the FECG in the IC's ECG. The $87.5 \%$ percentage potential for uterine contraction information suggest that the EMG of the IC's ECG can be obtained undistorted the majority of the time, but again improvements are needed during the occurrence of a modulation maternal ECG.
The final test for maternal ECG cancellation with clinical data consists of an objective measure for the expected value of maternal ECG suppression. Obtaining an accurate ratio of suppression is difficult. The difficulty comes from the inability to accurately determine the peak-to-peak value of the residual in the resulting IC's ECG from noise. Many of the suppression values were calculated using peak-to-peak values from noise since the maternal ECG residual(s) was difficult to distinguish. Since the masking noise was greater in amplitude than the maternal ECG residual, a conservative estimate has resulted when calculating the maternal ECG suppression ratio. From analyzing the suppression of 192 maternal ECG complexes from eight patients with various signal characteristics, the expected value was found to be $8.8 \mathrm{~V} / \mathrm{V}$. When considering that the FECG and maternal ECG are generally 
TABle 4: Percentage successes of the 10 test conditions.

\begin{tabular}{lcccccccccc}
\hline Test conditions & 1 & 2 & 3 & 4 & 5 & 6 & 7 & 8 & 9 & 10 \\
\hline Percentage successes & 100 & 75 & 87.5 & 100 & 100 & 100 & 100 & 100 & 100 & 0 \\
\hline
\end{tabular}

TABLE 5: Percentage potentials for the clinical applications.

\begin{tabular}{llc}
\hline Clinical applications & Percentage potentials \\
\hline 1 & $\begin{array}{l}\text { Fetal HR information via HR } \\
\text { determination algorithm }\end{array}$ & $87.5 \%$ \\
\hline 2 & $\begin{array}{l}\text { FECG electrophysiological } \\
\text { information via FECG averaging }\end{array}$ & $75 \%$ \\
\hline 3 & $\begin{array}{l}\text { Uterine contraction } \\
\text { information via EMG }\end{array}$ & $87.5 \%$ \\
\hline
\end{tabular}

similar in amplitude or the maternal ECG amplitude is less than the FECG, an expected suppression of $8.8 \mathrm{~V} / \mathrm{V}$ indicates that the maternal ECG attenuation algorithm performed effectively. The effectiveness is further reinforced when considering the expected suppression ratio was obtained under a variety of signal characteristics and using a conservative calculation method.

\section{CONCLUSION}

This study introduces a real-time method for maternal ECG cancellation of a FECG obtained via an IC. The method is based on maternal ECG averaging and subtraction and uses a thoracic ECG as a trigger to accurately perform the averaging and subtraction operations. Clinical data was obtained from eight patients to determine the methods effectiveness. The favorable results from the objective and subjective analyses indicate that the method for maternal ECG cancellation generally proved to be effective with synthesized and clinical data.

Since the three corruption measures for maternal ECG cancellation with synthesized data indicated no change to the resulting IC's ECG compared to the IC's ECG, these measures are considered favorable. Furthermore, since the FECG average from synthesized data contained 1\% of noise, was 0.995 correlated with the pure FECG complex, and had fully discernible P, QRS, and T waves, these results indicate that the corruption measures for maternal ECG cancellation and FECG SNR enhancement are also favorable. Therefore, the analysis verifies that the proposed method has performed effectively with synthesized data and has significant potential to enhance clinical data accurately.

For the subjective analysis of clinical data, Table 4 shows that eight of ten test conditions had percentage successes of $87.5 \%$ or greater, which indicates that the method was effective for those test conditions. However, improvements can still be made for a modulated maternal ECG of the IC's ECG as indicated by the low-percentage successes of test conditions 2 and 10. One possible solution for the resulting maternal ECG residual(s) from test condition 10 is to reduce the residual during FECG SNR enhancement. Reduction of the maternal ECG residual via FECG SNR enhancement could include FECG averaging as was performed in this study. Finally, the method's effectiveness for maternal ECG cancellation is further reinforced from the conservatively derived expected value of $8.8 \mathrm{~V} / \mathrm{V}$ for the maternal ECG suppression ratio.

The percentage potentials for the first two clinical applications of Table 5 were partially calculated based on a visual inspection for the presence or nonpresence of the FECG in the IC's ECG. Clinical applications 1 and 2 were included to give the reader percentages for the clinical data that had a visually detectable FECG. One of the eight patients studied had no noticeable FECG since the fetus had already significantly descended towards the pelvis area, which makes acquiring a FECG difficult as discussed in Section 2.2. In addition, another of the eight patients studied had a FECG that HR information could be determined but no electrophysiological information since the FECG was weak in amplitude again from significant fetal descent. However, similar maternal ECG cancellation was obtained with both these data set as with the six patient data sets that did have a noticeable FECG. This study is consistent with the finding of the first IC article which also reported reductions in the FECG amplitude when significant fetal descent has occurred [2]. Future research is necessary to determine a protocol for placement of the IC to obtain a FECG with a favorable SNR consistently. Clinical application 3 's percentage potential of $87.5 \%$ is another indicator of the proposed methods effective for maternal ECG cancellation. The reason the percentage of clinical application 3 was not closer to $100 \%$ is from the difficulty the method has with a modulated maternal ECG of the IC's ECG.

Since adaptive filtering has been applied to the noninvasive abdominal wall FECG data and IC ECG data and only maternal ECG averaging and subtraction has been applied to abdominal wall FECG data, this paper files a hole in the literature. In addition, this study is the first to analyze the effectiveness of maternal ECG cancellation with a FECG obtained via an IC. The first previously published IC method did not perform maternal ECG cancellation, but did indicate that 
signal processing was necessary. The reported IC and adaptive filter approach did perform maternal ECG cancellation, but did not perform a detailed subjective and/or objective analysis of the methods effectiveness with synthesized and clinical data. The researchers of the reported IC and adaptive filter technique limited the bandwidth of the IC's ECG to 15.0 to $40.0 \mathrm{~Hz}$ since the technique was used for fetal HR determination. For electrophysiological information of the FECG to be determined, the resulting IC's ECG after maternal ECG cancellation must have a diagnostic bandwidth of 0.05 to $100.0 \mathrm{~Hz}$ [8]. The proposed method is the first to report maternal ECG cancellation of an IC's ECG for a diagnostic bandwidth. Finally, a good future research topic would be to develop the reported IC and adaptive filter technique for a diagnostic bandwidth of 0.05 to $100.0 \mathrm{~Hz}$ and use a single thoracic ECG signal for maternal ECG cancellation of the IC's ECG. In addition, the future research could include a comparison of the modified IC and adaptive filter technique with the method proposed in this paper.

Finally, the FECG IC can be combined with the intrauterine pressure catheter to form one catheter versus the scalp electrode and intrauterine pressure catheter that are presently used for determining fetal status. Since the combined catheter is noninvasive to the fetus, the catheter could also be used in the presence of viral agent(s).

Future research is needed to determine a technique for placing the IC to consistently obtain a FECG that has a favorable SNR. This study did not focus on placement of the IC. When an effective technique is found for placing the IC, clinical trials of more than eight patients would then be appropriate to determine the effectiveness of the IC for obtaining a FECG with a favorable SNR and proposed maternal ECG cancellation technique for a large patient pool. The analyses for the effectiveness of maternal ECG cancellation of this paper could readily be expanded to a study of a larger patient pool.

\section{REFERENCES}

[1] A. C. Deans and P. J. Steer, "The use of the fetal electrocardiogram in labour," British Journal of Obstetrics and Gynecology, vol. 101, no. 1, pp. 9-17, 1994.

[2] N. J. Randall, P. J. Steer, and I. A. Sutherland, "Detection of the fetal ECG during labour by an intrauterine probe," Journal of Biomedical Engineering, vol. 10, no. 2, pp. 159-164, 1988.

[3] T. H. Strong Jr., R. H. Paul, G. D. Park, et al., "The intrauterine probe electrode," American Journal of Obstetrics and Gynecology, vol. 164, no. 5 (Pt 1), pp. 1233-1234, 1991.

[4] P. Bergveld, A. J. Kölling, and J. H. J. Peuscher, "Real-time fetal ECG recording," IEEE Transactions on Biomedical Engineering, vol. 33, no. 5, pp. 505-509, 1986.

[5] E. H. Hon and S. T. Lee, "Noise reduction in fetal electrocardiography II. Averaging techniques," American Journal of Obstetrics and Gynecology, vol. 87, pp. 1086-1096, 1963.

[6] V. D. Saini and D. Maulik, "Computerized detection of lowlevel fetal signals in the maternal abdominal electrocardiogram," Computers in Biology and Medicine, vol. 15, no. 2, pp. 81-93, 1985.

[7] B. Widrow and S. Stearns, Adaptive Signal Processing, Prentice-Hall, Englewood Cliffs, NJ, USA, 1985.
[8] A. Cohen, "Biomedical signals: origin and dynamic characteristic; frequency-domain analysis," in The Biomedical Engineering Handbook, J. D. Bronzino, Ed., pp. 805-827, CRC Press, Boca Raton, Fla, USA, 1995.

[9] B. Widrow, J. R. Glover, J. M. McCool, et al., "Adaptive noisecancelling: Principles and applications," Proceedings of the IEEE, vol. 63, no. 12, pp. 1692-1716, 1975.

[10] E. R. Ferrara and B. Widrow, "Fetal electrocardiogram enhancement by time-sequenced adaptive filtering," IEEE Transactions on Biomedical Engineering, vol. 29, no. 6, pp. 458-460, 1982.

[11] D. Adam and D. Shavit, "Complete foetal ECG morphology recording by synchronised adaptive filtration," Medical \& Biological Engineering \& Computing, vol. 28, no. 4, pp. 287-292, 1990.

[12] A. G. Favret and A. Marchetti, "Fetal electrocardiographic wave forms from abdominal-wall recordings," Obstetrics and Gynecology, vol. 27, no. 3, pp. 355-362, 1966.

[13] S. Cerutti, G. Baselli, S. Civardi, et al., "Variability analysis of fetal heart rate signals as obtained from abdominal electrocardiographic recordings," Journal of Perinatal Medicine, vol. 14, no. 6, pp. 445-452, 1986.

[14] S. Abboud, G. Barkai, S. Mashiach, and D. Sadeh, "Quantification of the fetal electrocardiogram using averaging technique," Computers in Biology and Medicine, vol. 20, no. 3, pp. 147-155, 1990.

[15] S. Abboud, A. Alaluf, S. Einav, and D. Sadeh, "Real-time abdominal fetal ECG recording using a hardware correlator," Computers in Biology and Medicine, vol. 22, no. 5, pp. 325$335,1992$.

[16] S. L. Horner and W. M. Holls, "Ultra-low-noise instrumentation and computer-based data acquisition and processing system for obtaining a noninvasive and intrauterine catheter fetal electrocardiogram," Critical Reviews in Biomedical Engineering, vol. 28, no. 1-2, pp. 165-171, 2000.

[17] M. S. Woolfson, W. Peasgood, D. S. Sahota, and J. A. Crowe, "Signal processing of the fetal electrocardiogram," Journal of Electrocardiology, vol. 23, pp. 51-57, 1990.

Steven L. Horner received the B.S., M.S., and Ph.D. degrees in electrical engineering from the University of Tennessee Knoxville in 1990, 1991, and 1996, respectively. From 1996 to 1998 , he was an Assistant Professor of electrical engineering at the University of Wyoming and since 1998 has been an Assistant Professor of electrical engineering at Bucknell University in Lewisburg, Pa., USA. His research interests include signal

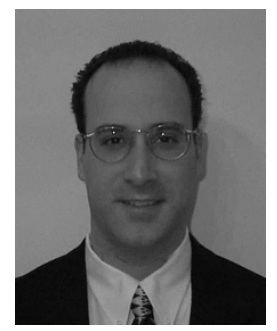
processing with application to biopotential signals, particularly, the noninvasive and invasive fetal electrocardiograms. Other interests include very large scale integrated circuits (VLSI) and field programmable gate array (FPGA) design.

William M. Holls III received the B.S. degree in physics from University of Notre Dame in 1967. He received an M.D. from Georgetown University School of Medicine in 1977, did his internship at Los Angeles County-USC Medical Center from 1977-1981, and his fellowship in Maternal-Fetal medicine at Georgetown University from 1981 to 1983 . He is presently a Clinical Associate Professor of Obstetrics and Gynecology at the University of Illinois. Present research interests include fetal physiology, noninvasive and invasive fetal assessment, and computer applications in medicine. 